\title{
A Lightweight Chaos-Based Medical Image Encryption Scheme Using Random Shuffling and XOR Operations
}

\author{
Fawad Masood ${ }^{1} \cdot$ Maha Driss $^{2,3} \cdot$ Wadii Boulila $^{2,3} \cdot$ Jawad Ahmad $^{4}$. \\ Sadaqat Ur Rehman ${ }^{5}$ Sana Ullah Jan ${ }^{6} \cdot$ Abdullah Qayyum $^{7}$. William J. Buchanan ${ }^{4}$
}

Accepted: 4 May 2021 / Published online: 19 May 2021

(C) The Author(s) 2021

\begin{abstract}
Medical images possess significant importance in diagnostics when it comes to healthcare systems. These images contain confidential and sensitive information such as patients' $\mathrm{X}$-rays, ultrasounds, computed tomography scans, brain images, and magnetic resonance imaging. However, the low security of communication channels and the loopholes in storage systems of hospitals or medical centres put these images at risk of being accessed by unauthorized users who illegally exploit them for non-diagnostic purposes. In addition to improving the security of communication channels and storage systems, image encryption is a popular strategy adopted to ensure the safety of medical images against unauthorized access. In this work, we propose a lightweight cryptosystem based on Henon chaotic map, Brownian motion, and Chen's chaotic system to encrypt medical images with elevated security. The efficiency of the proposed system is proved in terms of histogram analysis, adjacent pixels correlation analysis, contrast analysis, homogeneity analysis, energy analysis, NIST analysis, mean square error, information entropy, number of pixels changing rate, unified average changing intensity, peak to signal noise ratio and time complexity. The experimental results show that the proposed cryptosystem is a lightweight approach that can achieve the desired security level for encrypting confidential image-based patients' information.
\end{abstract}

Keywords Lightweight cryptosystem · Henon chaotic map · Chen's chaotic system · Brownian motion $\cdot$ Cryptographic technique $\cdot$ Medical image

Jawad Ahmad

j.ahmad@napier.ac.uk

1 College of Information Engineering, Yangzhou University, Yangzhou 225127, China

2 RIADI Laboratory, University of Manouba, 2010 Manouba, Tunisia

3 College of Computer Science and Engineering, Taibah University, Medina 42353, Saudi Arabia

4 School of Computing, Edinburgh Napier University, Edinburgh, EH10 5DT, UK

5 Department of Computer Science, Namal Institute, Mianwali, Pakistan

6 University of the West of Scotland, Paisley, UK

7 Department of Electrical Engineering, University of Engineering and Technology, Peshawar, 25120, Pakistan 


\section{Introduction}

The telemedicine is a fast growing field of providing medical care for patients remotely where the patient and the healthcare provider are not physically present at the same location. The patient's confidential information such as medical images are shared through communication channels of Internet or cellular network. Such a modern healthcare system demands efficient framework capable of storing medical images such that they are always accessible only to authorized users present at any geological position. The cloud storage systems can provide such a platform, however, these systems are vulnerable to cyber attacks particularly when designed below security standards. Nevertheless, the researchers have largely focused on designing computer-based strategies for improving patient care. However, the techniques to achieve the desired level of security of the confidential information in both of the storage systems and the communication channels has lagged somewhat. In this situation, one way of protecting the medical images is through encryption schemes where the images are encrypted in such a way that they become useless for the users with no encryption information in hand. Some of the security solution for telemedicine applications are discussed in [13,32].

The digital images communication in medicine (DICOM) is recognized as an international standard (ISO 12052) for medical imaging in modern electronic healthcare system. There are various DICOM tools and systems designed for diagnostic purposes in pre-procedural medical analysis. Such systems are highly dependent on signal processing to visualize internal organs of the human body. Moreover, today's medical operations are mostly controlled by artificial intelligence (AI) enabled systems with integrated sensors. These real-time decision making systems, that sometimes even collaborate in sensitive surgical operations, produce as well as depend on sensitive medical data which is generally in the form of digital images. Usually, to protect these medical images that contain sensitive information about patients requires a robust system with real-time safeguarding techniques. In fact, the e-health systems, just like ordinary cloud storage systems, are vulnerable to various types of attacks, therefore, storing the medical images in its original form are comparatively easy to access by breaching system's security protocols only. Moreover, the management and transmission of medical data between different cloud systems adapted by different hospitals or medical centers increases this vulnerability. As a result, an efficient, robust, and computationally agile system is needed to safeguard sensitive medical images against attacks irrespective of the storage systems and the communication protocols [8].

The data security systems can be divided into two types: hiding information and encrypting information. The hiding information is further classified into steganography and watermarking. The steganography tries to cover or mask the data such that the existence of data is concealed during sharing. On the other hand, in cryptography, the encripted sensitive data is visible publicly, however, the values are obscure to the viewers. Furthermore, watermarking relies on embedding a unique identification watermark to the raw data. Here, the watermarked signature is extracted at the receiving end. The aim is to prevent illegal exploitation of copyrighted or confidential contents. Besides, the concept of information encryption is to convert the plain data (original contents) into some indecipherable form before transmission. The objective is to preserve the contents in a way that it can be recovered by authorized users through decryption. The image encryption is achieved in such a way, i.e, by distorting each pixel of the image [19, 21, 22, 34].

Chaos-based cryptography is an innovative domain that employs various chaotic maps to generate random sequencing for digital medical image encryption $[1,6,28,36,42,43$, 
46]. These simple chaotic maps exhibit unique properties including non-linearity (NL), sensitivity towards initial conditions, strange attractors with different initial keys, and randomness. These properties can be used in order to design a robust cryptosystem [27, 29, 30, 33, 40, 41].

The initial conditions of the system are very important as they are the secret keys that should be known only to authorized persons. Without having information about these keys, the data can not be decrypted by anyone. In fact, initial conditions of the system are sensitive particularly because hackers and unauthorized users usually apply a minute change to try and decrypt the secured content. The high sensitive system means that changing small values will display profoundly strange attractors and as a result, hackers will be incapable of decrypting the original content. In addition, the strength of any cryptosystem is assessed by its computational complexity, while the transmission rate determines the efficiency of the whole system.

Claude Shannon originally introduced the copulated properties of confusion (substitution) and diffusion (permutation) in 1949 [38]. When coupled, these properties assisted in generating random numbers. Later, random numbers were applied to encrypt digital images. These copulated properties of confusion and diffusion can permute (swap) the pixel position of an image or substitute (change the pixels' values), either way, providing high-level security. Early researchers and cryptographers in the field utilized one or the other to design cryptosystems, however, cryptosystems with partial strengths were not sufficient to provide adequate security. Therefore, cryptographers started incorporating different features of confusion and diffusion that resulted in improved security $[9,26]$. The subsequent phase of diffusion is based on pixel distortion, i.e., adding an additional layer of protection to the initial phase of confusion and thus improving overall security.

In general, two principal components are necessary to design a secure scheme; the image encryption symmetric key and the private/public key. In a symmetric key-based cryptosystem the authorized user uses the same key at both ends. This type of security is widely adopted for computer security. The single key is shared between two or more users i.e., use to convert plain text to ciphertext and cipher text to plain text back. In Fig. 1, the symmetric key is applied to the chaos-based cryptographic algorithm that assisted to change the plain image into the encrypted image. In the next stage, the same key is applied to the encrypted image resulting in decryption process. In subsequent Fig. 2 the plain data is converted to ciphered data using encryption key $K 1$. By applying key $K 1$ the data is transformed into ciphered form. Furthermore the ciphered data is decrypted in the second

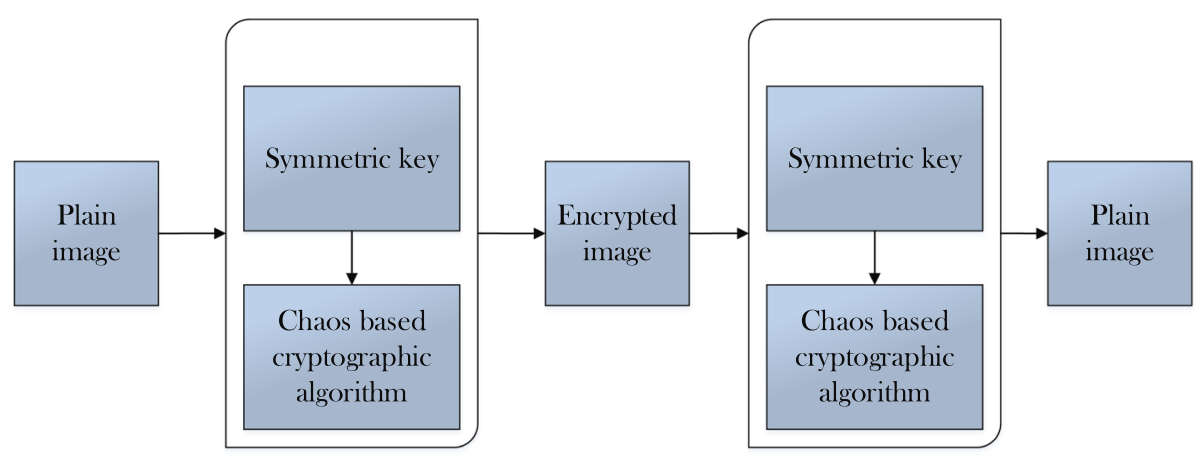

Fig. 1 Schematic chart for symmetric key based encryption and decryption 


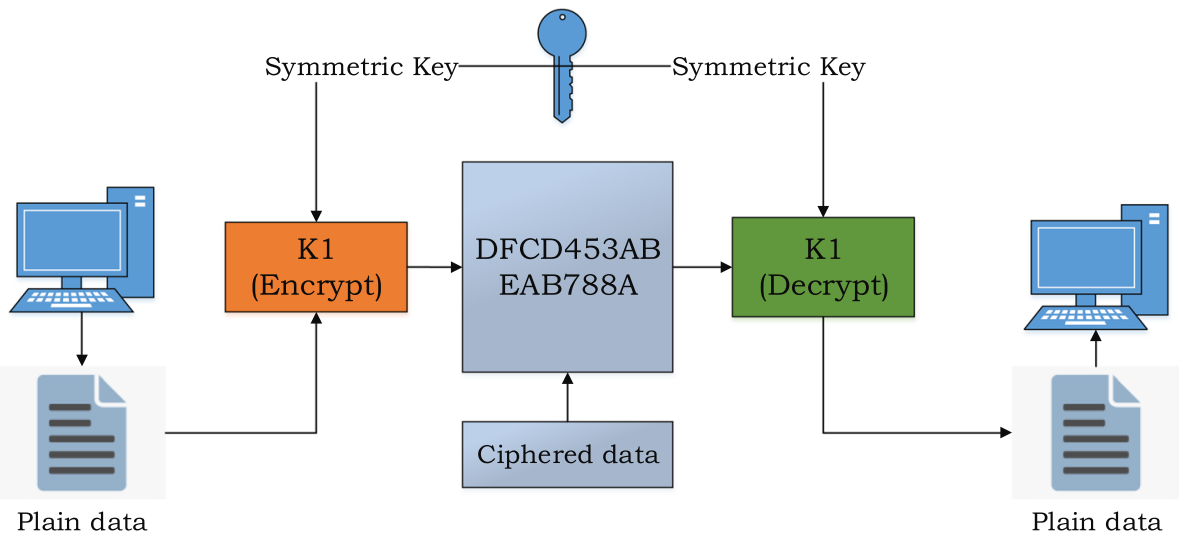

Fig. 2 Another view of symmetric key based encryption and decryption process

phase using same key $K 1$ resulting in getting back plain data at the other end. Systematic charts of two different symmetric key-based encryption and decryption methods are shown in Figs. 1 and 2.

\section{Literature Review}

A secure cryptography scheme can be achieved through different ways, including permutation and substitution, as shown in Fig. 3. Over the last decade, researchers have proposed a number of image encryption schemes using chaos theory [1, 2, 9]. Nevertheless, hybrid cryptosystems are emerging as more promising solution due to their efficiency in encrypting multimedia information $[10,14]$. Existing hybrid systems use different domains and multiple substitution or permutation processes to shuffle and distort the pixels of an image. For instance, chaotic maps are famous for producing dynamic and unpredictable responses in return. Given the sheer variation in initial condition, these chaotic maps produce drastic output and highly random sequencing that are difficult to decrypt without having specific

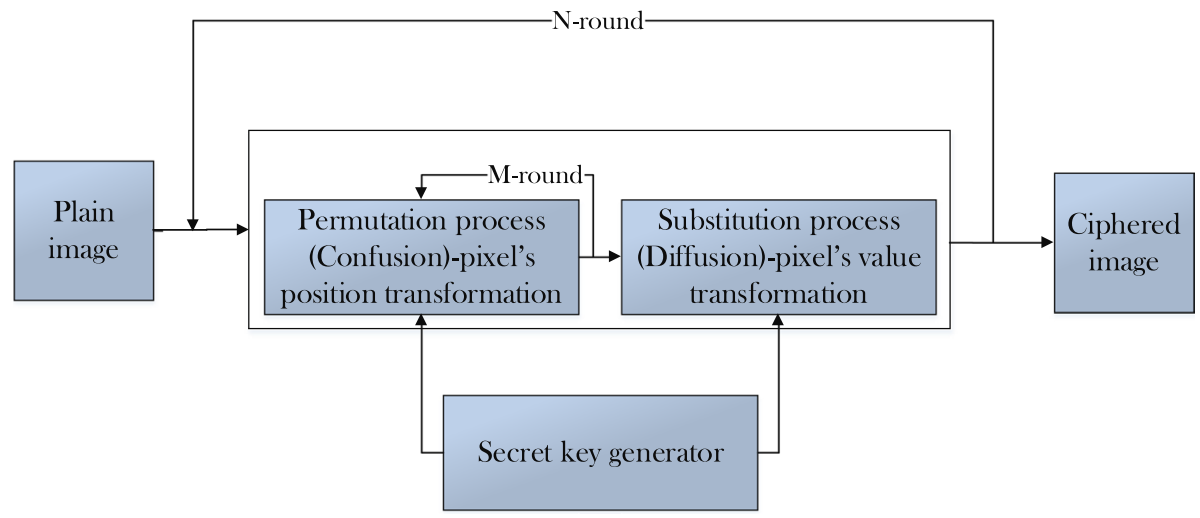

Fig. 3 Pixel's permutation and substitution with round process 
knowledge of the system. Therefore, these maps are widely used for image encryption to achieve secure communication [39].

Most of the researchers and cryptographers adapted such approaches to design cryptosystems with significant computational security. For instance, [31] proposed a cryptosystem based on the external key having two chaotic maps to generate random numbers. The external key emerges from the algorithm's initial state and is altered infrequently in order to encrypt each block cipher that comprises a total of sixteen pixels. It is very difficult for attackers to discover the secret key that has been used in the proposed method. Similarly, [11] designed a hybrid scheme of a chaotic map and a coupled map lattice. A single dimension chaos map is used, however, the combined effect is an improved key space ultimately improving protection across the entire system profoundly sensitive to its initial condition. In another study, [15] proposed a system by adapting a hyperchaotic map to depreciate prediction times. This cryptosystem includes permutation of pixels via shuffling matrix along with diffusion of the shuffled pixels. This system provides improved security due to the high key space associated with it.

\section{Contributions}

The major contribuions of this work are summarized as follows:

- Designing an effective multi-stage cryptographic algorithm for medical images encryption using substitution-permutation technique. This multi-stage cryptographic algorithm uses random numbers generated from chaos maps which reduces correlation among the pixels of the digital medical images.

- Designing a contemporary variant of the chaos-based confusion-diffusion approach that is capable of achieving a significant higher entropy and NIST-based randomness results as compared to existing methods. The results demonstrate that the proposed encryption algorithm is able to generate highly secured medical encrypted images.

- Analysing the performance of the proposed system and comparing it with several existing approaches used in cryptosystems. The efficiency of each algorithm is analyzed in terms of histogram consistency analysis and its variance (HCAV), adjacent pixels correlation analysis (APCA), contrast analysis (CA), homogeneity analysis (HA), energy analysis (EA), NIST analysis, information entropy (IE), a number of pixels changing rate (NPCR), unified average changing intensity (UACI), mean square error (MSE), peak to signal noise ratio (PSNR), and time complexity (TC).

\section{Proposed Algorithm}

This section presents detailed discussion about the proposed algorithm that uses multiple chaotic maps.

\subsection{Henon Chaotic Map (HCM)}

The Henon chaotic map (HCM), sometimes termed a Henon-Pomeau attractor map [17], is a dynamic system of the discrete domain and one of the most reviewed examples of twodimensional dynamic structures that exhibit unpredictable/chaotic behaviours. The Henon 
map functions by taking any point along the plane $\left(x_{n}, y_{n}\right)$ and mapping it to a new one, a process that can be formulated as follows:

$$
\left\{\begin{array}{l}
x_{n+1}=1-a x_{n}^{2}+y_{n} \\
y_{n+1}=b x_{n}
\end{array}\right.
$$

As demonstrated above, this type of chaotic map relies on parameters $a$ and $b$. Moreover, this system is established for the value set to $a=1.4$ and $b=0.3$. A Henon map is chaotic in terms of traditional values, but may prove to be either chaotic, intermittent, or else converging to aperiodic orbit for other values of the same parameters. An overview of the Henon map's behaviour and form at the different values for its parameters can be obtained from its orbit diagram. The two-dimensional chaotic map is plotted for 10,000 iterations with the initial conditions set to $a=1.4$ and $b=0.3$ as shown in Fig. 4 .

\subsubsection{Brownian Motion (BM)}

Brownian motion is a spontaneous movement of particles floating in a liquid or gas substance due to interactions between the fast-moving atoms and molecules. The particles' evolution along three primary directions (here, $\mathrm{X}, \mathrm{Y}$, and Z) and mathematically defined as [23]:

$$
X=r \sin a \cos b, \quad Y=r \sin a \sin b, \quad Z=r \cos a
$$

whereas $0 \leq r \leq+\infty, 0 \leq b \leq 2 \pi$, and $0 \leq a \leq \pi$

The state of the Brownian particle can be measured when sufficient information is related to the direction of motion of the particles, i.e., particles' movement along three directions (X, $\mathrm{Y}$, and $\mathrm{Z}$ ). The specific time duration $\left(t_{p}\right)$ is the time needed for the particles to move erratically, the total number of particles $\left(n_{p}\right)$ involved in the zig-zag motion, and the number of impulses per change in track associated with a zig-zag motion. The step length is signified as $r=2$ and the pseudo-random function is utilized to determine the direction of movement of particles. In this way, the X, Y, and Z attributes of each Brownian particle's position can be obtained. BM can be generated using the Monte Carlo process. In three-dimensional model,

Fig. 4 Two-dimensional chaotic map for 1000 iterations with initial conditions $a=1.4$ and $b=0.3$

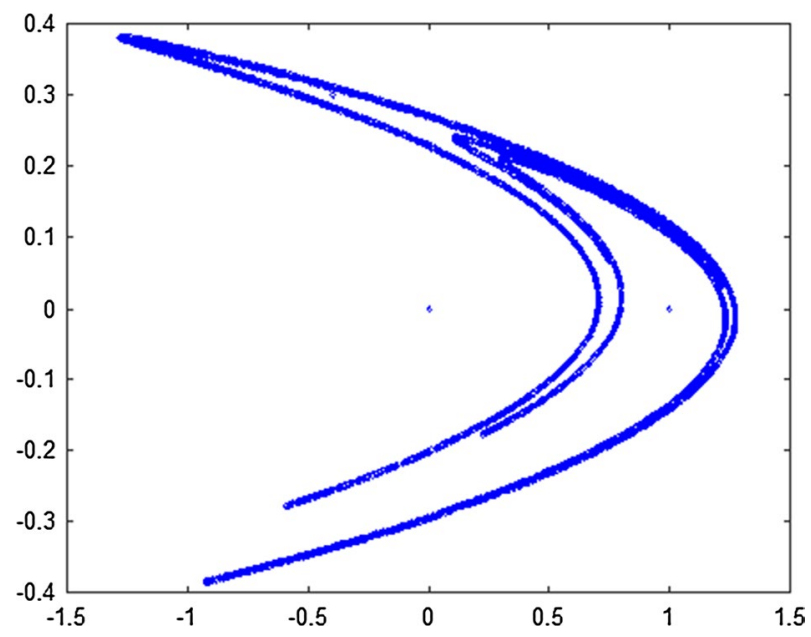


we estimate the Brownian motion of 10 particles, using a vector of $10 \times 3$ to store Brownian particles' impulse per change in track, particle positions, and distance. The plot diagram for all the three directions is shown in Fig. 5. The proposed scheme uses zig-zag random numbers for all the three directions generated for number of particles $(n p)=256$, total estimated time $(t)=$ $60 \mathrm{~s}$, and number of impulses per change in track $=\mathrm{N}=100 \times t($ where: $\mathrm{t}=60 \mathrm{~s})$.

\subsubsection{Chaotic Chen System (CSS)}

The chaotic system based on [12] is defined as follows:

$$
\begin{aligned}
& \frac{\mathrm{d} x}{\mathrm{~d} t}=a(y-x) \\
& \frac{\mathrm{d} y}{\mathrm{~d} t}=(c-a) x-x z+c y \\
& \frac{\mathrm{d} z}{\mathrm{~d} t}=x y-b z
\end{aligned}
$$

Here $a, b$, and $c \in R^{3}$ are fixed. If $a=35, b=3$, and $c=35$. then the system involves a chaotic attractor, as can be seen in Fig. 6.

The fractional order of this particular system may be defined as:

$$
\begin{aligned}
\frac{\mathrm{d}^{q} x}{\mathrm{~d} t^{9}} & =a(y-x) \\
\frac{\mathrm{d}^{q} y}{\mathrm{~d} t^{q}} & =(c-a) x-x z+c y \\
\frac{\mathrm{d}^{q} z}{\mathrm{~d} t^{q}} & =x y-b z
\end{aligned}
$$

where $q$ reflects a fractional order with a specific range of $0<q \leq 1$. Therefore, we change only the derivative order $q$ and the system parameter $c$ in this simulation process while
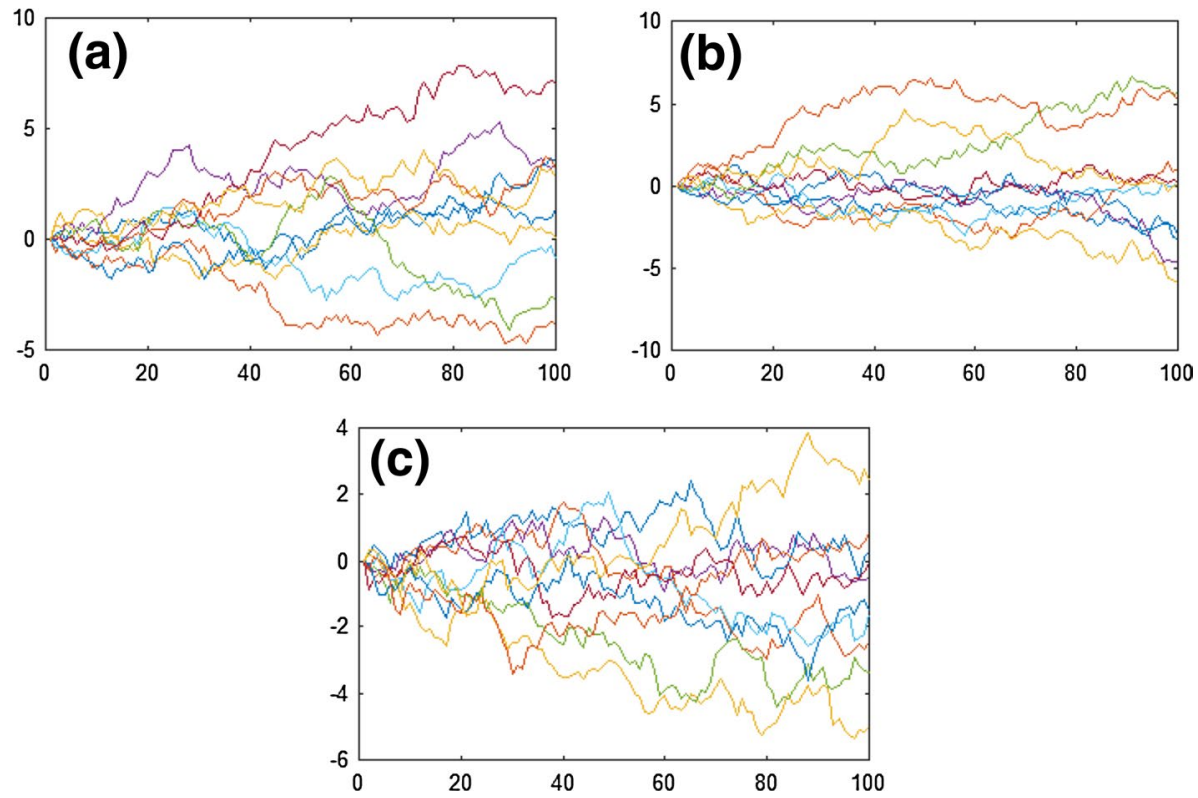

Fig. 5 Brownian motion particles along $\mathrm{X}(\mathbf{a}), \mathrm{Y}(\mathbf{b})$, and $\mathrm{Z}(\mathbf{c})$ directions for $n p=10, t=10 \mathrm{~s}$, and N/T $=10$ 
Fig. 6 3D view of CCM attractor within a fractional-order Chen system (4) with $q=0.1$ while ( $a$, $b, c)=(35,3,28)$

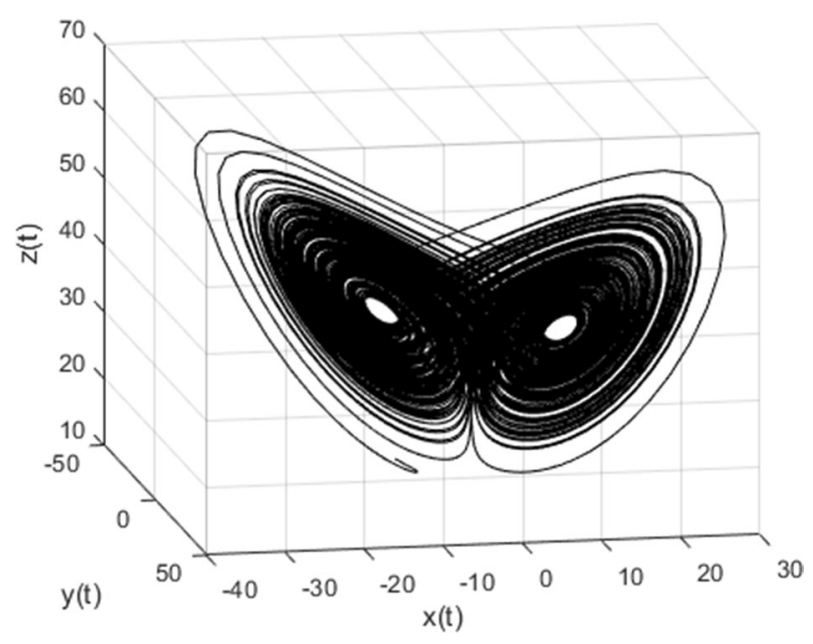

the rest of the input variables remain constant. Notice that both $q$ and $c$ varied as shown in [24]. Simulations are conducted for a step size of 0.1 with $q=0.6-0.1$. Here, the simulation results indicate that, with an order beyond 3, chaos tends to exist. While $q=0.6-0.1$ is in the time step 0.1, there are also chaotic attractors, as the process shown in Fig. 6 demonstrates. However, if the value of $q=0$, then no chaotic behaviour is perceived, implying that $q=0-0.1$ is the lowest fractional-order $q$ limit at which it is possible for chaos to exist in this kind of system (4). 0.3 was the lowest order at which we discerned chaos.

\subsection{The Proposed Algorithm}

The flow chart of the proposed medical image encryption scheme is shown in Fig. 7. From the flow chart, one can see that, several essential steps are required in order to develop a cryptosystem secure enough to encrypt medical images given as follows:

(1) Let $I$ be the plain text image with $m \times n$ representing the entire dimension of a grey medical image:, $m$ and $n$ represent the image's rows and columns, respectively. Each medical image $I$ is resized to a dimension of $512 \times 512$ pixels and is stored as $I_{2}$.

(2) The resized image $I_{2}$, which now contains 262144 pixels, is further divided into an equal number of blocks, i.e., $I_{2}=B_{1}, B_{2}, B_{3}, B_{4} \ldots B_{4096}$, in which the total number of blocks is 4096 and each block size is $8 \times 8$. An example of this step is shown in Fig. 8 .

(3) In third step, a two-dimensional HCM is initiated and utilized to shuffle the $8 \times 8$ pixels of each generated block, thus producing an intra-block shuffling process. An example of this step is shown in Fig. 9.

(4) After the intra-block shuffling process, image blocks are shuffled up to third phase as shown in Fig. 10.

(5) Next, a three-dimensional BM is initiated to define the number of particles concerning the time in seconds, the defining position of various particles, and the number of impulses per each change made in each of three directions (X, Y, and Z): e.g., $X=T_{1}$, $Y=T_{2}$, and $Z=T_{3}$. These particles are then stored in each direction, where the total number of particles $(n p)=256$, the total time $(t)=60$, and the number of impulses per change in track $=N=100 . * t($ where: $\mathrm{t}=60 \mathrm{~s})$. 


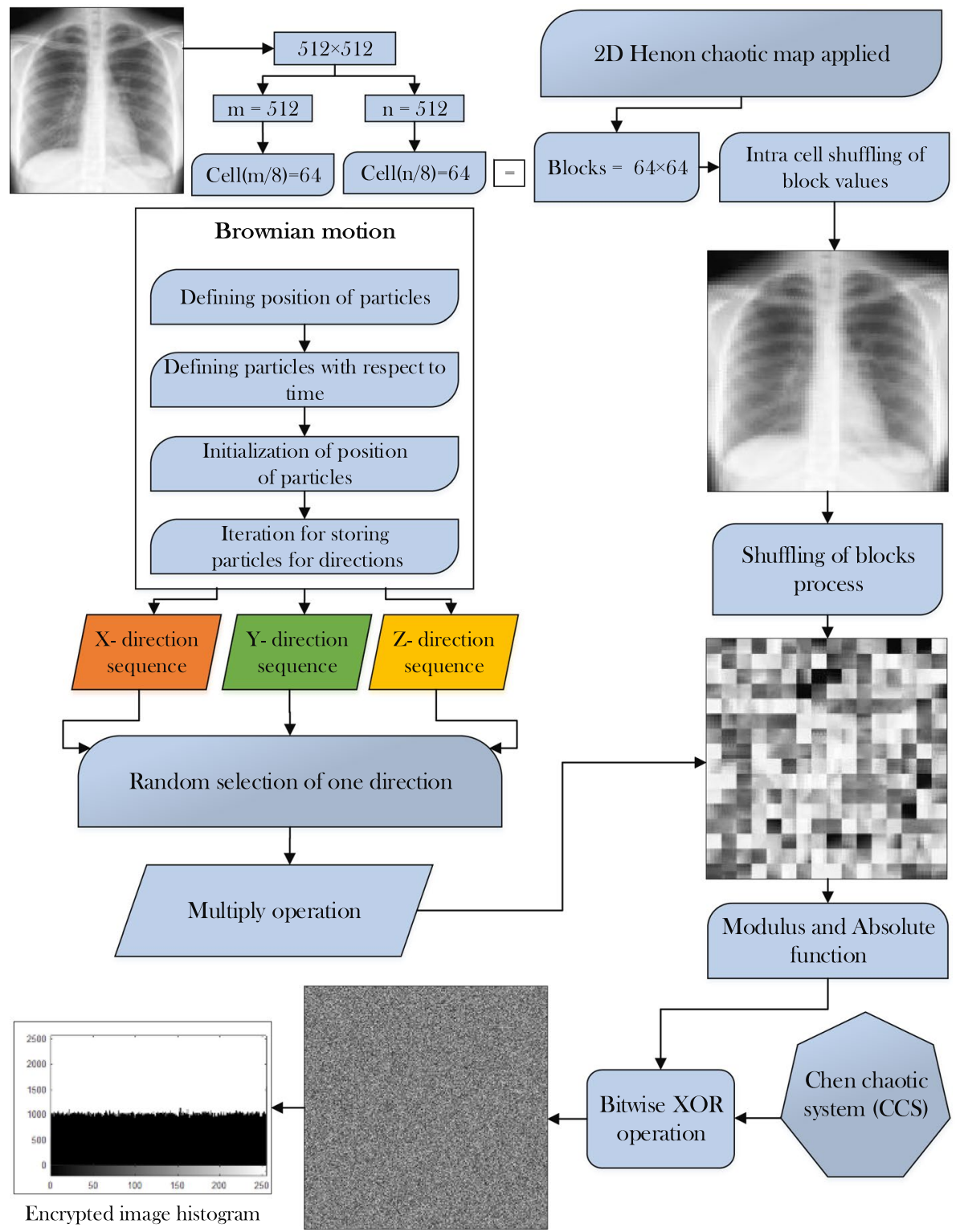

Encrypted image

Fig. 7 Flow chart for the proposed medical cryptosystem

(6) In this step, BM is iterated $N=1536256$ times for each direction $T_{1}, T_{2}$, and $T_{3}$, respectively. The first 1274112 values are discarded, i.e., 1536256-1274112 = 262144 from each direction, as a means of overcoming the transient effect. Some of the random values thus generated are then stored in $U_{1}, U_{2}$, and $U_{3}$, respectively. 


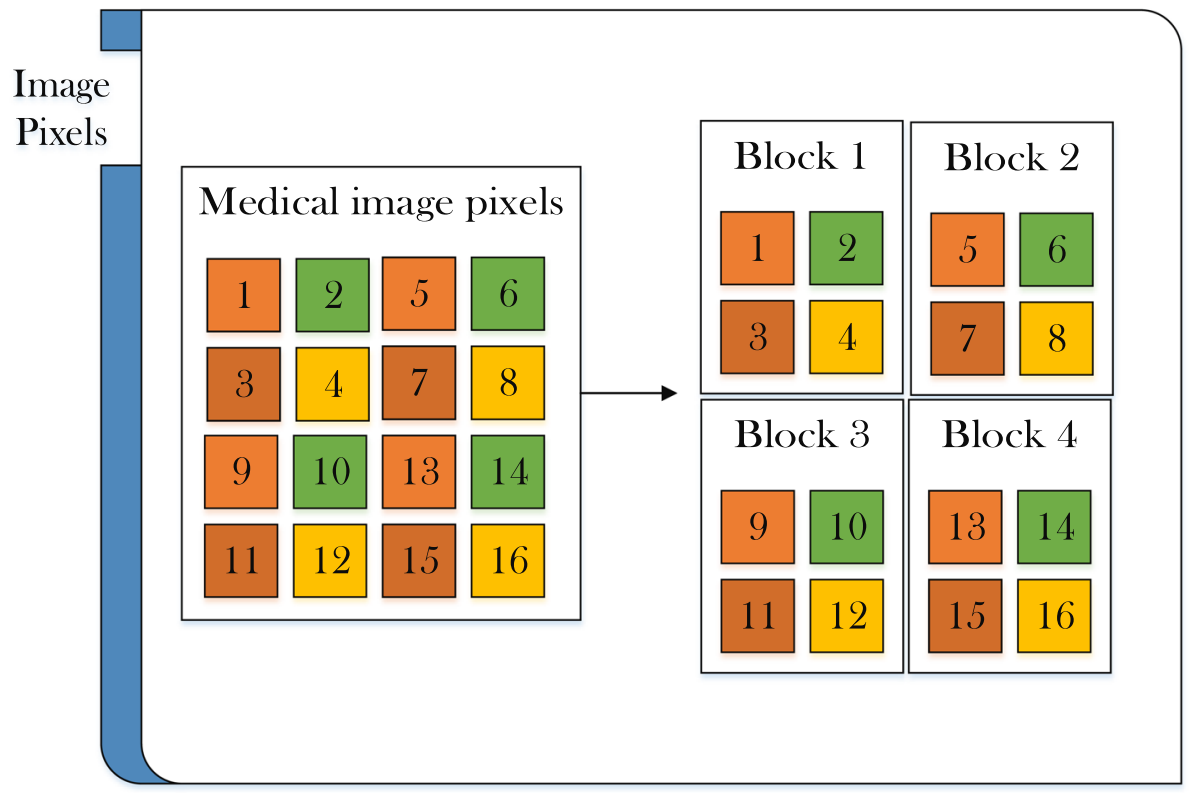

Fig. 8 Initial phase of pixels distribution into No. of blocks $=4096$

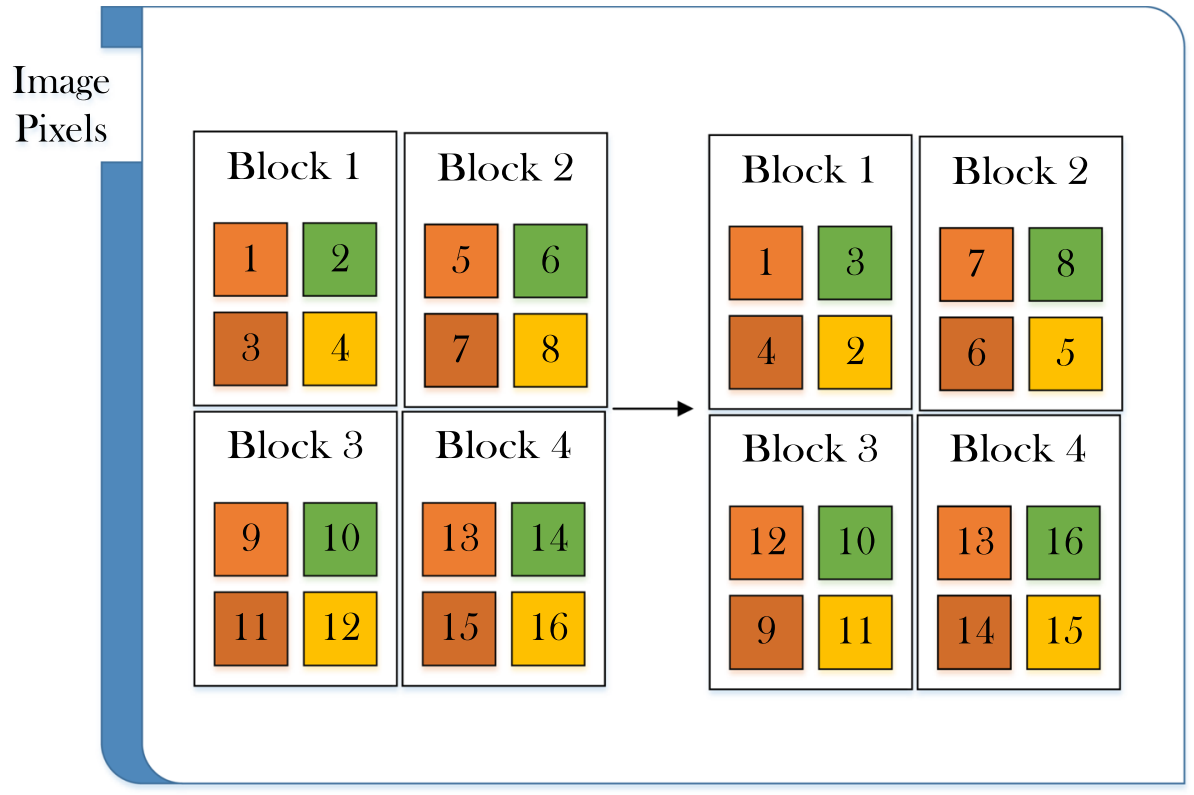

Fig. 9 Second phase of pixels shuffling inside each block

(7) The values of $U_{1}, U_{2}$, and $U_{3}$ are now multiplied by $10^{14}$ to get $V_{1}, V_{2}$, and $V_{3}$, respectively. Moreover $V_{1}, V_{2}$, and $V_{3}$ are now reshaped to a matrix of $512 \times 512$ pixels, 


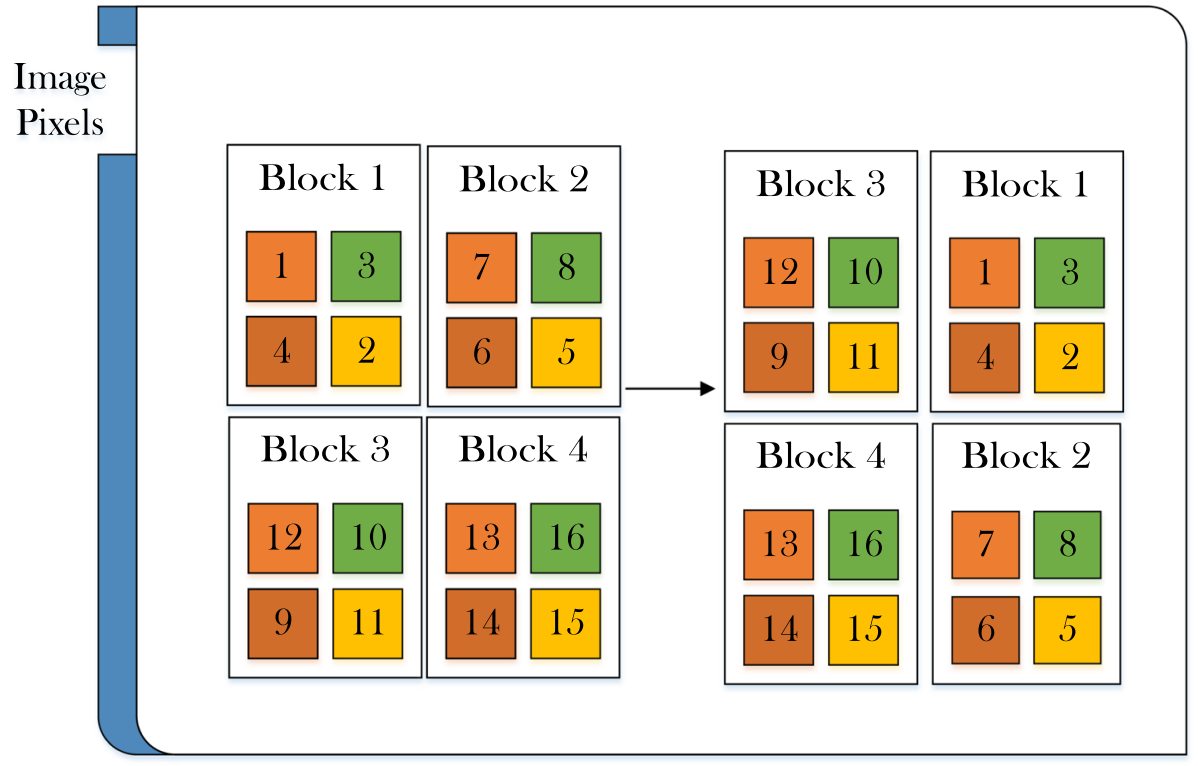

Fig. 10 Third phase of itself blocks shuffling

which helps achieve random sequencing for three directions: $W_{1}, W_{2}$, and $W_{3}$.

(8) Absolute and round function are applied to $W_{1}, W_{2}$, and $W_{3}$ and the new values are stored in $X_{1}, X_{2}$, and $X_{3}$, respectively.

(9) Modulus 256 operation is applied to $X_{1}, X_{2}$, and $X_{3}$ in order to get $Y_{1}, Y_{2}$, and $Y_{3}$.

(10) Zig-zag random sequencing $Y_{1}, Y_{2}$, and $Y_{3}$ is multiplied with the permuted grey image of third phase $I_{3}$ (step 4) and the new values are stored in $Z_{1}, Z_{2}$, and $Z_{3}$.

(11) Fractional-order CCS is added for an additional layer of security increasing the randomness and entropy of the proposed scheme.

(12) Finally, the most recent output of the CCS random sequencing is bitwise XOR with $Z_{1}, Z_{2}$, and $Z_{3}$ to get new encrypted layers such as $Z 1_{\text {encrypt }}, Z 2_{\text {encrypt }}, Z 3_{\text {encrypt }}$.

\section{Experimental Results}

The performance of the proposed scheme is evaluated using a number of tests which are widely used to assess the statistical measures and security of cryptosystems. The tests for performance analysis of proposed scheme are conducted on a core i5 CPU with 4GB RAM.

\subsection{Histogram Analysis}

All numerical values repeated in an image can be visually observed with the help of a histogram. The histogram of an image should have a uniform distribution and must not have 
sharp peaks. The uniform distribution of an encrypted image on a histogram shows the higher security and efficiency of the encryption.

The proposed scheme is tested on various grey medical images, including Chest, Brain, and MR and the results are displayed in Figs. 11, 12, 13, 14, 15, 16, 17, 18, 19 and 20. The pixels are permuted (shuffled) during the first phase of the proposed scheme, achieving the outputs shown in Figs. 11, 12 and 13. Each medical image is encrypted along with three directions, as shown in Figs. 14, 15 and 16. Histograms of each medical image in these three directions are shown in Figs. 17, 18, 19 and 20. In Fig. 17 pixels are not uniformly distributed while in Figs. 18, 19 and 20, each pixel of encrypted image is uniformly distributed along three different directions. Thus the results of this test have validated our
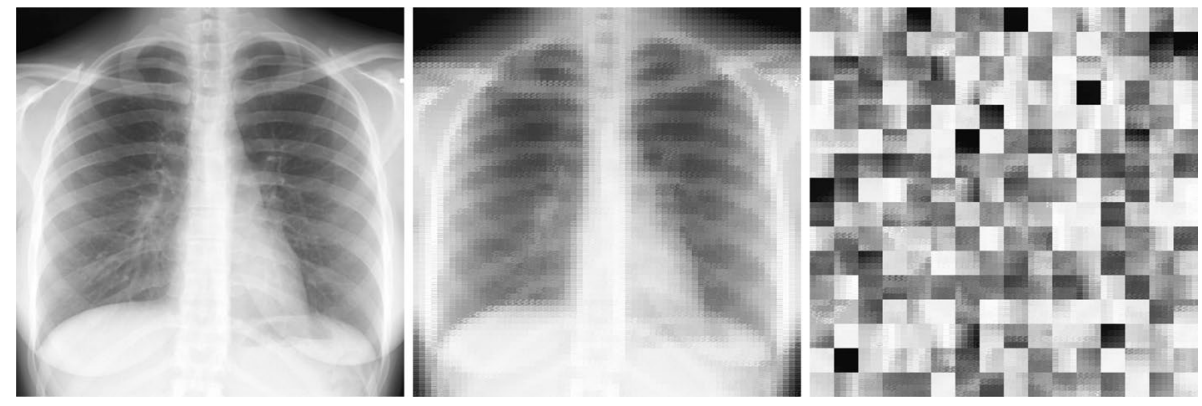

Fig. 11 Chest X-ray Original image, shuffling of block values, shuffling of blocks themselves
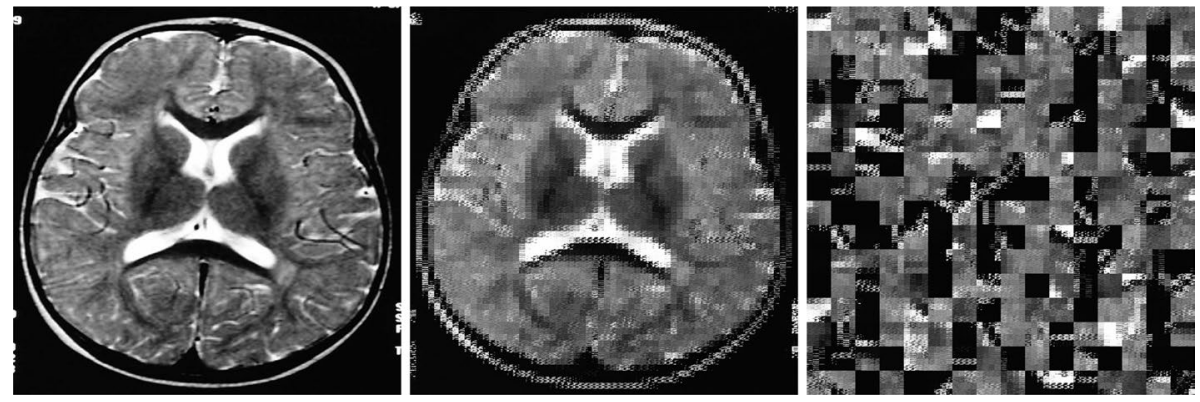

Fig. 12 Brain image Original image, shuffling of block values, shuffling of blocks themselves
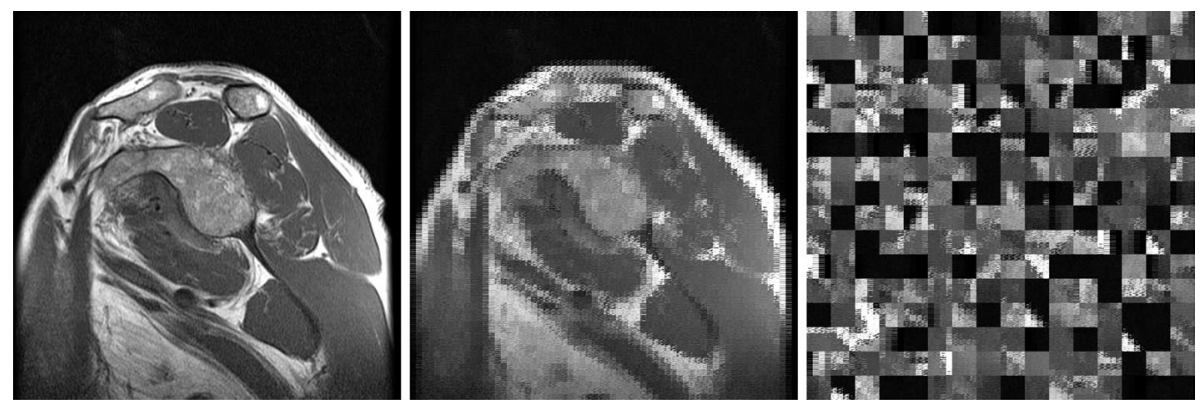

Fig. 13 MR image Original image, shuffling of block values, shuffling of blocks themselves 

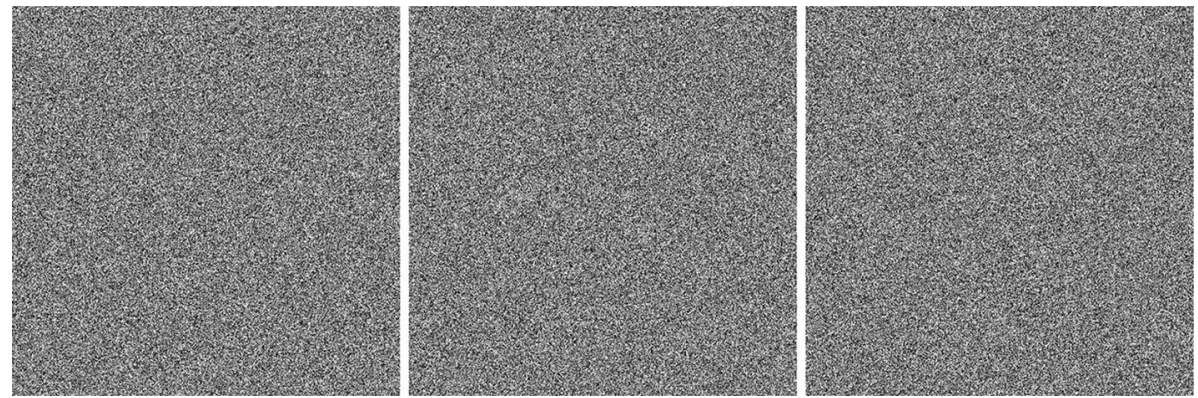

Fig. 14 Chest $X$-ray Encrypted image along X encrypted image along Y, encrypted image along Z
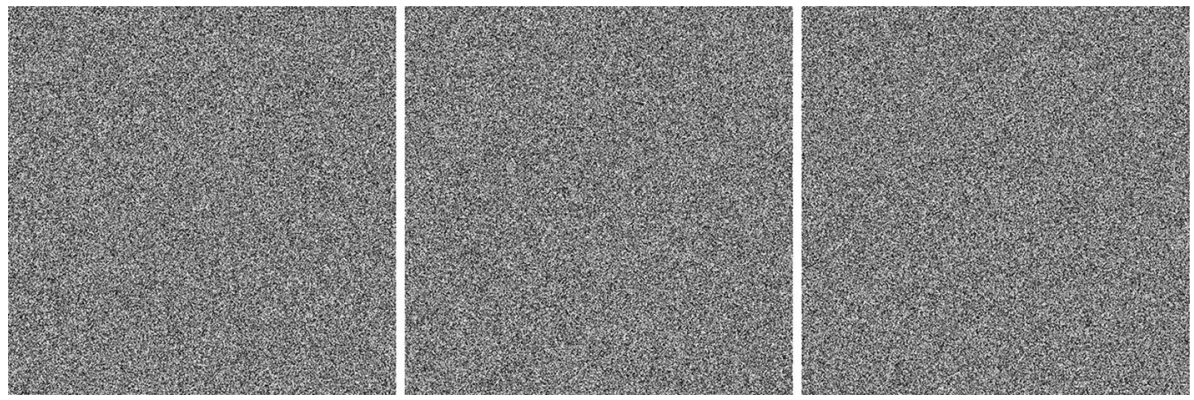

Fig. 15 Brain image Encrypted image along X, encrypted image along Y, encrypted image along Z
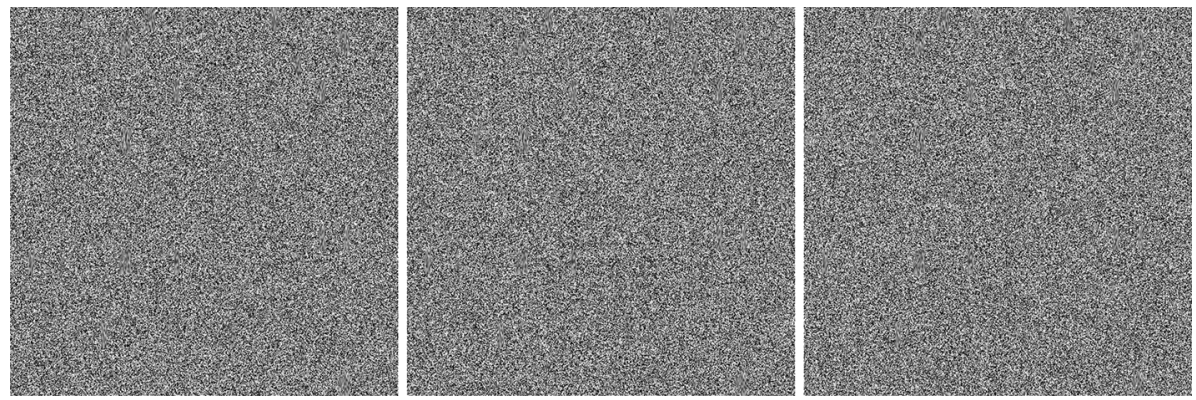

Fig. 16 MR image Encrypted image along X, encrypted image along Y, encrypted image along Z
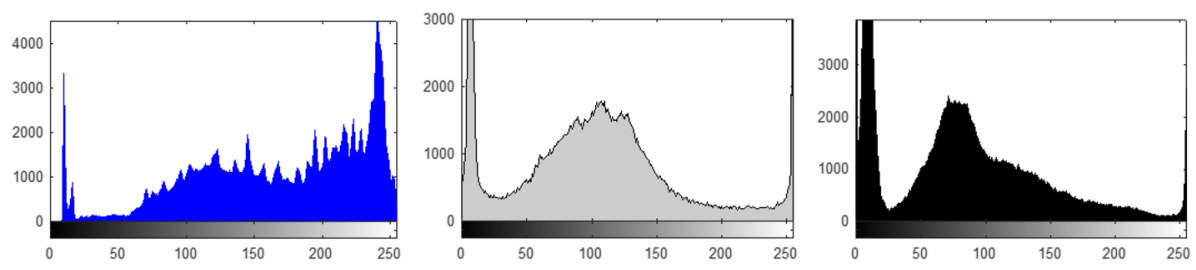

Fig. 17 Plain image histograms Chest X-ray histogram, Brain image histogram, MR image histogram 

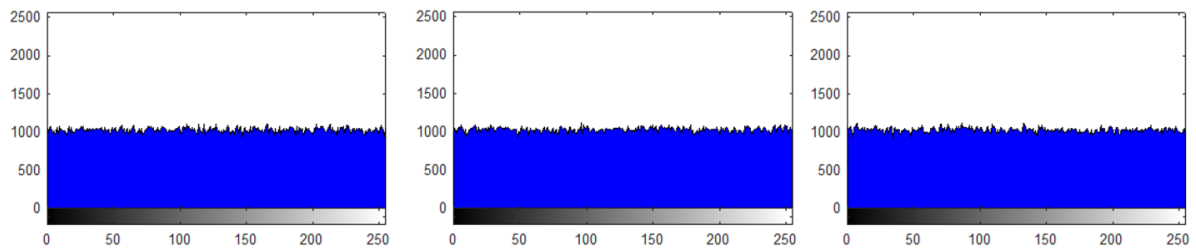

Fig. 18 Encrypted chest $X$-ray image histograms encrypted along X, encrypted along $\mathrm{Y}$, encrypted along Z
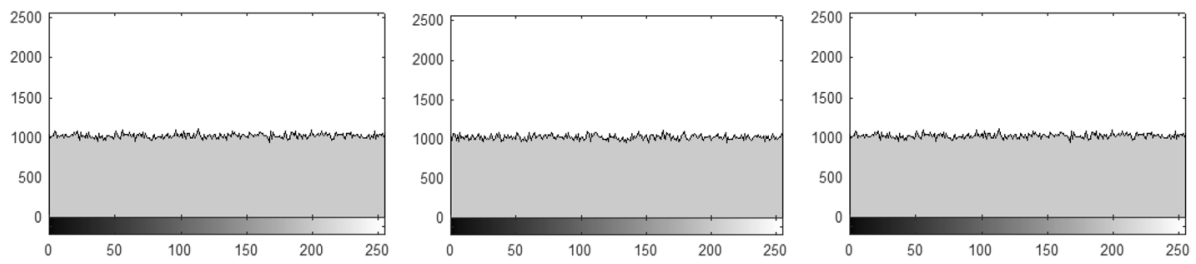

Fig. 19 Encrypted brain image histograms encrypted along X, encrypted along Y, encrypted along Z
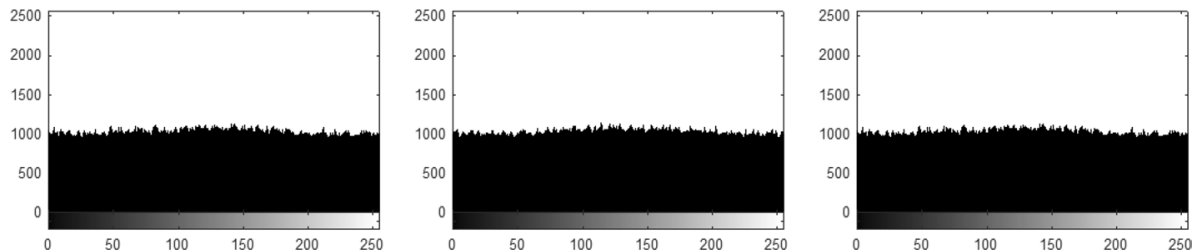

Fig. 20 Encrypted MR image histograms encrypted along X, encrypted along Y, encrypted along Z
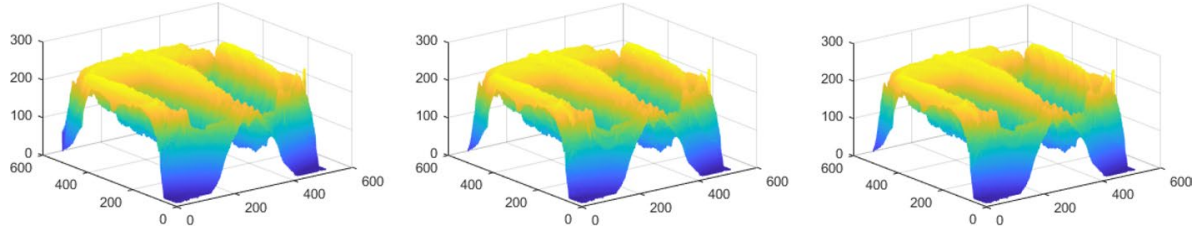

Fig. $213 D$ view plain image histograms Chest X-ray histogram, Brain image histogram, MR image histogram

suggested cryptosystem (Fig. 21). Furthemore, three dimensional (3D) view of plaintext and encrypted histogram is also shown in Figs. 22, 23, 24 and 25. One can also confirm from Figs. 22, 23, 24 and 25 that the histogram of encrypted image is flat as compared to plaintext image.

\subsection{Adjacent Pixels Correlation Analysis}

An important parameter to demonstrate the diffusion and confusion properties of a ciphertext image is correlation among adjacent pixels. In this work, a total of four thousand 

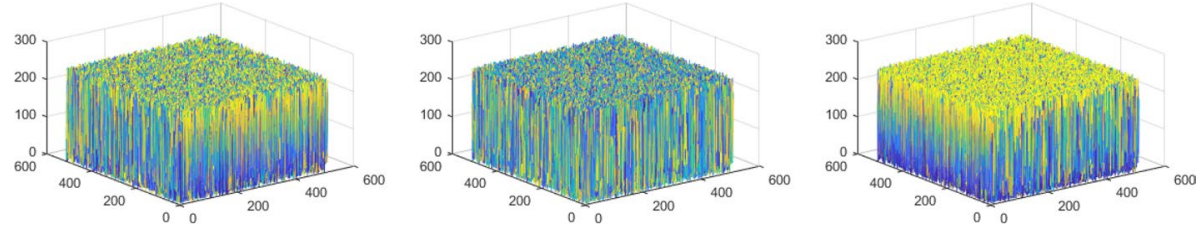

Fig. $223 D$ view chest image histograms Chest X-ray histogram along X horizontally, Chest X-ray histogram along $\mathrm{X}$ diagonally, Chest $\mathrm{X}$-ray histogram along $\mathrm{X}$ vertically
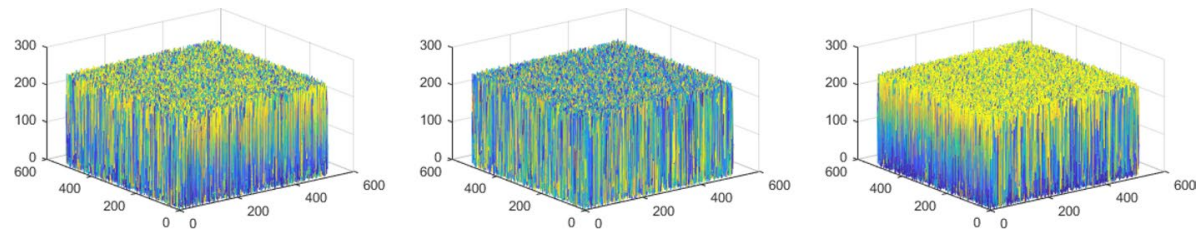

Fig. $233 D$ view chest image histograms Chest X-ray histogram along Y horizontally, Chest X-ray histogram along Y diagonally, Chest X-ray histogram along Y vertically
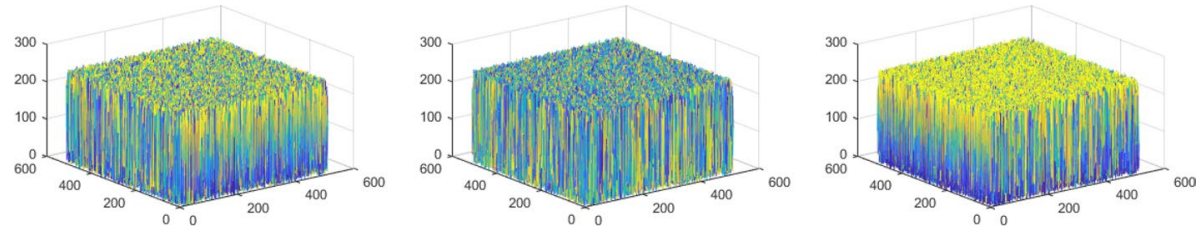

Fig. $243 D$ view chest image histograms Chest X-ray histogram along Z horizontally, Chest X-ray histogram along $\mathrm{Z}$ diagonally, Chest $\mathrm{X}$-ray histogram along $\mathrm{Z}$ vertically

random pairs of pixels adjacent horizontally, diagonally, and vertically from both the plaintext and the ciphertext versions of the same image were analyzed. Mathematically, correlation can be calculated as:

$$
\begin{gathered}
r_{x y}=\frac{E((x-E(x))(y-E(y)))}{\sqrt{D(x) D(y)}} \\
E(x)=\frac{1}{N} \sum_{i=1}^{N} x_{i} \\
D(x)=\frac{1}{N} \sum_{i=1}^{N}\left(x_{i}-E(x)\right)^{2} .
\end{gathered}
$$

where $x$ and $y$ represent the grey-scale values of adjacent pixels and $E(x)$ is the expected mean value. The range of correlation coefficient is between -1 to 1 , where 1 illustrate the exact similarity between two images or pixels. It is essential to obtain a value near 0 for the case of maximum uncorrelated pixels, i.e., highly random values. The pixel similarity or dissimilarity between plaintext and ciphertext for different medical images is explored and 
Table 1 Correlation coefficient values for each dimension

\begin{tabular}{|c|c|c|c|c|c|c|c|c|c|}
\hline \multirow{2}{*}{\multicolumn{2}{|c|}{ Images }} & \multicolumn{4}{|c|}{ Plain image dimensions } & \multicolumn{4}{|c|}{ Encrypted image dimensions } \\
\hline & & H-D & D-D & V-D & $\mathrm{A}-\mathrm{V}$ & H-D & D-D & V-D & $\mathrm{A}-\mathrm{V}$ \\
\hline 1 & Chest- $X$ direction & 0.9988 & 0.9975 & 0.9985 & NA & 0.0023 & -0.0008 & 0.0007 & NA \\
\hline 2 & Chest-Y direction & 0.9988 & 0.9975 & 0.9985 & NA & -0.0012 & 0.0021 & -0.0002 & NA \\
\hline 3 & Chest- $Z$ direction & 0.9988 & 0.9975 & 0.9985 & NA & -0.0035 & 0.0016 & 0.0003 & NA \\
\hline 4 & Brain-X direction & 0.9831 & 0.9566 & 0.9713 & NA & 0.0000 & -0.0030 & -0.0029 & NA \\
\hline 5 & Brain-Y direction & 0.9831 & 0.9566 & 0.9713 & NA & 0.0014 & 0.0002 & -0.0009 & NA \\
\hline 6 & Brain- $Z$ direction & 0.9831 & 0.9566 & 0.9713 & NA & -0.0004 & 0.0020 & 0.0002 & NA \\
\hline 7 & MR-X direction & 0.9850 & 0.9783 & 0.9881 & NA & 0.0021 & -0.0016 & 0.0065 & NA \\
\hline 8 & MR-Y direction & 0.9850 & 0.9783 & 0.9881 & NA & 0.0010 & 0.0007 & 0.0058 & NA \\
\hline 9 & MR-Z direction & 0.9850 & 0.9783 & 0.9881 & NA & 0.0020 & -0.0022 & 0.0050 & NA \\
\hline 6 & [5] & 0.9727 & 0.9204 & 0.9573 & - & -0.0394 & -0.0194 & -0.0223 & - \\
\hline 7 & [35] & - & - & - & - & 0.0681 & 0.0128 & 0.0049 & - \\
\hline 8 & [25] & - & - & - & - & 0.0965 & 0.0362 & -0.0581 & - \\
\hline 9 & [18] & - & - & - & - & 0.1257 & 0.0226 & 0.0581 & - \\
\hline
\end{tabular}

Here, H-D = Horizontal dimension, $\mathrm{D}-\mathrm{D}=$ Diagonal dimension, and $\mathrm{V}-\mathrm{D}=$ Vertical dimension, $\mathrm{A}-\mathrm{V}=$ Cumulative average value
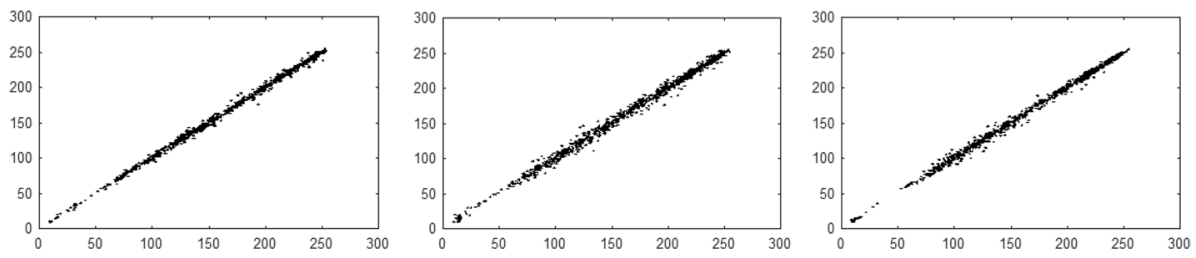

Fig. 25 Plaintext chest image pixels correlation Chest X-ray pixel's correlation along X, Chest X-ray pixel's correlation along Y, Chest X-ray pixel's correlation along Z

compared with $[5,18,25,35]$. The results of our proposed system are given in Table 1 . The visual results given in Fig. 25 reflect all the three directions of the grey scale chest X-ray image where pixels are joined diagonally. These results demonstrate that for the plaintext, image pixels remain highly similar. The pixels are diffused in all directions when the pixels of encrypted images have zero resemblance to one other as shown in Figs. 26, 27, and 28. In Table 1 the dimension wise plain image mean values of Chest, Brain, and MR for each direction $\mathrm{X}, \mathrm{Y}$ and $\mathrm{Z}$ are approximately $0.9982,0.9703$, and 0.9838 , respectively that is near to 1 which corresponds to high correlation between pixels of an image for each direction. The mean values are calculated in case of encrypted image of Chest, Brain, and MR for each direction. The calculated encrypted average or mean values are $0.0015,-0.0034$, and 0.0019 . The values lies in between 0 and -1 for encrypted image. These results validate our proposed cryptosystem.

\subsection{Homogeneity, Energy and Contrast Analyses}

The proximity of grey-level co-occurrence matrices (GLCM) elements in this system can be quantified by homogeneity analysis. Statistical combinations of pixel luminosity or 

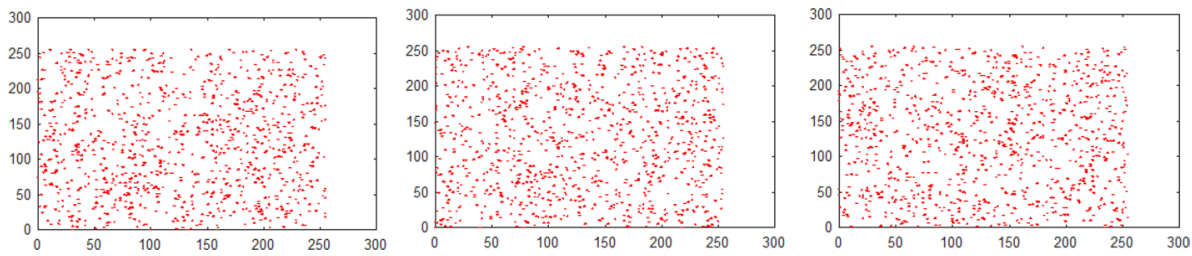

Fig. 26 Encrypted chest image pixels correlation Chest X-ray pixels correlation along X horizontally, Chest $\mathrm{X}$-ray pixels correlation along X diagonally, Chest X-ray pixels correlation along X vertically
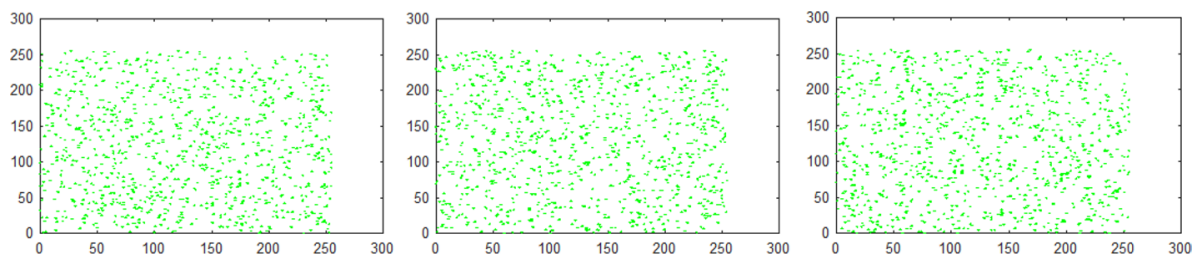

Fig. 27 Encrypted chest image pixels correlation Chest X-ray pixels correlation along Y horizontally, Chest $\mathrm{X}$-ray pixels correlation along Y diagonally, Chest X-ray pixels correlation along Y vertically
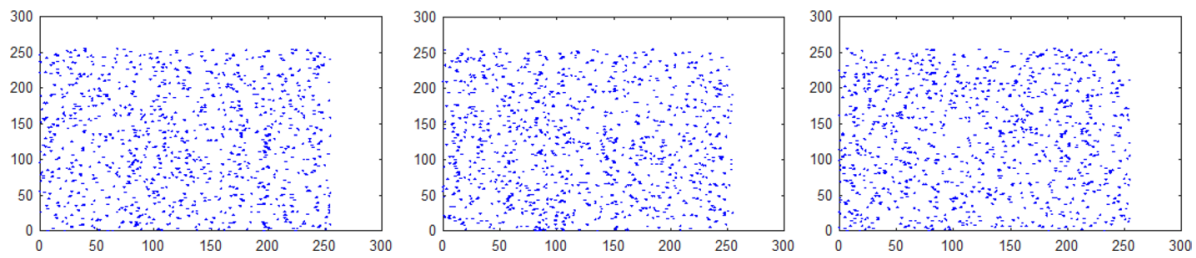

Fig. 28 Encrypted chest image pixels correlation Chest X-ray pixels correlation along Z horizontally, Chest $\mathrm{X}$-ray pixels correlation along $\mathrm{Z}$ diagonally, Chest $\mathrm{X}$-ray pixels correlation along $\mathrm{Z}$ vertically

grey levels is illustrated by GLCM tables. Here, if the homogeneity values are lower, the encryption scheme is considered efficient. Mathematically, this can be calculated as:

$$
H=\sum_{x, y=1}^{M} \frac{g(x, y)}{1+|x-y|} .
$$

where $g(x, y)$ stands for the grey-level co-occurrence matrices in GLCM.

Contrast is the disparity in luminosity or colours by which the items in a picture can be differentiated and viewers can recognise various objects. The difference in the intensity of adjacent pixels across the entire image can be computed with contrast analysis. For increased security, the contrast values should be higher, which demonstrates the amount of randomness in the ciphertext image. The mathematical expression for contrast is:

$$
\text { Contrast }=\sum_{i, j=1}^{M}|x-y|^{2} p(x, y) .
$$

where $p(x, y)$ signifies the grey-level co-occurrence matrices in GLCM. 
Table 2 Average homogeneity, energy, and contrast analysis along $\mathrm{X}$ direction

\begin{tabular}{lllll}
\hline S. no. & Algorithms & Homogeneity & Energy & Contrast \\
\hline 1 & Chest & 0.3890 & 0.0156 & 10.5305 \\
2 & Brain & 0.3895 & 0.0156 & 10.5081 \\
3 & MR & 0.3933 & 0.0157 & 10.2801 \\
\hline
\end{tabular}

\begin{tabular}{lllll}
\hline S. no. & Algorithms & Homogeneity & Energy & Contrast \\
\hline 1 & Chest & 0.3894 & 0.0156 & 10.4844 \\
2 & Brain & 0.3888 & 0.0156 & 10.5022 \\
3 & MR & 0.3936 & 0.0157 & 10.2778 \\
\hline
\end{tabular}

Table 3 Average homogeneity, energy, and contrast analysis along $\mathrm{Y}$ direction
Table 4 Average homogeneity, energy, and contrast analysis along $\mathrm{Z}$ direction

\begin{tabular}{lllll}
\hline S. no. & Algorithms & Homogeneity & Energy & Contrast \\
\hline 1 & Chest & 0.3899 & 0.0156 & 10.4780 \\
2 & Brain & 0.3894 & 0.0156 & 10.4716 \\
3 & MR & 0.3932 & 0.0157 & 10.2948 \\
\hline
\end{tabular}

Table 5 Existing results of homogeneity, energy, and contrast analysis

\begin{tabular}{lllll}
\hline S. no. & Algorithms & Homogeneity & Energy & Contrast \\
\hline 1 & [20]-Pepper & 0.4644 & 0.0210 & 7.7123 \\
2 & [4]-Pepper & 0.9455 & 0.2133 & 0.2219 \\
\hline
\end{tabular}

Energy is another parameter that can be calculated from the GLCM. In this case, the energy analysis measures squared elements. The mathematical expression for energy calculation is as follows:

$$
\text { Energy }=p(\boldsymbol{x}, \boldsymbol{y})^{2} .
$$

where $p(x, y)$ signifies the overall number of grey-level co-occurrence matrices.

Homogeneity values for the test images of the Chest, Brain, and MR images are depicted in Tables 2, 3 and 4. The obtained average value of homogeneity for the proposed scheme is 0.3906 for $\mathrm{x}, \mathrm{y}$, and $\mathrm{z}$ direction of each medical image. Moreover, the output of proposed scheme is compared with the schemes proposed by $[4,20]$, and the results are illustrated in Table 5. The values generated by the proposed scheme are comparatively flat compared to the results in Table 5. Therefore, the low values obtained demonstrate the efficiency of our proposed cryptosystem.

Energy values of ciphertext images for chest X-ray, brain, and MR medical images are also shown in Tables 3 and 4. The calculated average value for the suggested scheme is 0.0156. Moreover, the outputs/results achieved with our proposed cryptosystem are compared to those obtained with $[4,20]$, as presented in Table 5. The proposed scheme values are comparatively low to those calculated in Table 5, and hence, the examined results have authenticated our proposed cryptographic scheme. Moreover, from Tables 2, 3 and 4, the 
values of contrast can be also observed for ciphertext images in each of the three directions. The calculated average value is higher relative to existing schemes, such as $[4,20]$, as presented in Table 5. The proposed scheme values are comparatively high to those calculated in. Therefore it is evident that the proposed scheme possesses better resistance to attacks than many present schemes.

\subsection{NIST Analyses}

The National Institute of Standards and Technology (NIST), a US organization, issued specific guidelines that should be followed to secure data. Fifteen tests are proposed by the NIST as a means of estimating an encryption algorithm's randomness and measuring its strength. The performance of our proposed scheme is benchmarked in Table 6, which reveals that the proposed system has passed all fifteen tests recommended by the NIST and hence suggests that it is secure enough for digital encryption of medical images.

\subsection{Differential Attack Analysis}

Immunity to differential attacks is also a vital feature of an encryption algorithm. Two tests that can determine resistance to differential assaults are: (1) Number of pixels changing rate and (2) Unified average changing intensity. Each of the aforementioned tests is discussed in detail below, where both were run upon two encrypted images whose corresponding plaintext images differ from each other by a single pixel.

Table 6 Building type, scope, scale, privacy issues, sampling time and accuracies of various occupancy techniques

\begin{tabular}{|c|c|c|c|c|c|c|c|}
\hline \multirow[t]{2}{*}{ Test } & \multicolumn{6}{|c|}{$p$ values for grey medical Chest image along three direction } & \multirow[t]{2}{*}{ Results } \\
\hline & & Plain & $\mathrm{X}$ direction & Y direction & $\mathrm{Z}$ direction & & \\
\hline Frequency & & $3.5568 \times 10^{-07}$ & 0.72795 & 0.48662 & 0.65797 & Success & \\
\hline Block frequency & & $4.9729 \times 10^{-09}$ & 0.80617 & 0.82067 & 0.53435 & Success & \\
\hline Rank & & 0.29191 & 0.29191 & 0.29191 & 0.29191 & Success & \\
\hline Runs $(M=10,000)$ & & 0.75417 & 0.97325 & 0.35506 & 0.75417 & Success & \\
\hline Long runs of ones & & 0.035752 & 0.035752 & 0.035752 & 0.035752 & Success & \\
\hline $\begin{array}{l}\text { Overlapping tem- } \\
\text { plates }\end{array}$ & & 0.85988 & 0.85988 & 0.85988 & 0.85988 & Success & \\
\hline $\begin{array}{l}\text { No overlapping } \\
\text { templates }\end{array}$ & & 0.99999 & 0.99995 & 0.93985 & 0.99999 & Success & \\
\hline Spectral DFT & & 0.66336 & 0.77167 & 0.77167 & 0.66336 & Success & \\
\hline $\begin{array}{l}\text { Approximate } \\
\text { entropy }\end{array}$ & & 0.39444 & 0.26251 & 0.85323 & 0.39444 & Success & \\
\hline Universal & & 0.99931 & 0.99574 & 0.98619 & 0.99931 & Success & \\
\hline Serial & $p$ values 1 & 0.70248 & 0.37715 & 0.22973 & 0.70248 & Success & \\
\hline Serial & $p$ values 2 & 0.49286 & 0.12465 & 0.21782 & 0.49286 & Success & \\
\hline $\begin{array}{l}\text { Cumulative sums } \\
\text { forward }\end{array}$ & & 0.27679 & 0.24206 & 0.23771 & 0.27679 & Success & \\
\hline $\begin{array}{l}\text { Cumulative sums } \\
\text { reverse }\end{array}$ & & 0.77056 & 1.1217 & 0.85465 & 0.77506 & Success & \\
\hline
\end{tabular}




\subsubsection{Number of Pixels Changing Rate}

The number of changing pixels in two cipher text images can be calculated via the NPCR test when there is a minute difference of one pixel between their plaintext images. The mathematical formula of NPCR is:

$$
N P C R=\frac{\sum_{i, j} D(i, j)}{M \times N} \times 100 \%
$$

If the two ciphertext images has same value then $D(i, j)=0$ while in the opposite case, $D(i, j)=1$. The upper limit of the NCPR is $100 \%$, but for a a good cryptosystem, the NCPR value should be higher than $99.5 \%$.

\subsubsection{Unified Average Changing Intensity}

The degree of averaged changed intensity between two cipher text images can be calculated with the help of UACI test when there is a one-pixel difference between their corresponding plaintext images. The mathematical expression for UACI is:

$$
U A C I=\frac{1}{M \times N}\left[\sum_{i, j} \frac{\left|C_{1}(i, j)-C_{2}(i, j)\right|}{255}\right] \times 100 \%
$$

$C_{1}(i, j)$ and $C_{2}(i, j)$ signify the encrypted images whose corresponding plaintext images are different from each other by a single pixel. The values of NCPR and UACI can be observed from Table 7. The proposed scheme values are compared to that of [37], as shown in Table 8 . The values imply that our proposed cryptographic scheme offers much higher security relative to both modern and traditional cryptosystems.

\subsection{Pixel's Inconsistency Analysis}

\subsubsection{Mean Square Error}

Mean Square Error (MSE) is the measurement of the average of the squares of errors between two images and can be used to analyze Avalanche effect. According to this security metric, a substantial change in the encrypted image should result when a small

Table 7 NPCR and UACI calculated value for chest, brain, and MR image along three direction

\begin{tabular}{lllll}
\hline S. no. & Images & Direction & NPCR values & UACI values \\
\hline 1 & Chest & X-direction & 99.62 & 33.65 \\
2 & Chest & Y-direction & 99.63 & 33.60 \\
3 & Chest & Z-direction & 99.63 & 33.54 \\
4 & Brain & X-direction & 99.60 & 33.61 \\
5 & Brain & Y-direction & 99.62 & 33.59 \\
6 & Brain & Z-direction & 99.59 & 33.64 \\
7 & MR & X-direction & 99.62 & 33.36 \\
8 & MR & Y-direction & 99.61 & 33.27 \\
9 & MR & Z-direction & 99.60 & 33.32 \\
\hline
\end{tabular}


Table 8 Comparison of NPCR and UACI values

\begin{tabular}{lllll}
\hline S. no. & Images & Direction & NPCR values & UACI values \\
\hline 1 & Chest & X-direction & 99.62 & 33.65 \\
2 & Chest & Y-direction & 99.63 & 33.60 \\
3 & Chest & Z-direction & 99.63 & 33.54 \\
4 & {$[37]$} & - & 99.60 & 33.55 \\
5 & {$[37]$} & - & 99.60 & 33.41 \\
\hline
\end{tabular}

change is introduced in either the plaintext image or the key. The mathematical expression for MSE is as follows:

$$
\operatorname{MSE}=\frac{1}{W \times H} \sum_{i=1}^{W} \sum_{j=1}^{n}(\mathrm{X}(i, j)-\mathrm{Y}(i, j))^{2},
$$

where $W$ and $H$ illustrates the image's dimensions, while $X$ and $Y$ represents the couple of encrypted images themselves whose corresponding encryption keys are only different from each other by a single bit. The measured value of MSE should be high for any robust cryptosystems. We applied the MSE test on all three medical images for three different directions. The average MSE value (in all directions) for chest image, brain, and MR image is 11017, 12320, and 11468 respectively, as shown in Table 9. The proposed scheme assessed values are also compared to the present scheme of [44], demonstrated in Table 10. The higher value obtained by our proposed scheme showed that it is extremely secure, even in comparison to the most modern cryptosystems.

\subsubsection{Peak to Signal Noise Ratio}

This ratio gauges the change in pixel value between a plaintext image and its encrypted counterpart. For the calculation of PSNR, the plaintext image is chosen as a signal and the encrypted image as noise. Mathematically, PSNR is calculated as:

Table 9 Calculated MSE and PSNR values

\begin{tabular}{llllllll}
\hline S. no. & Algorithms & X-direction & Y-direction & Z-direction & X-direction & Y-direction & Z-direction \\
\hline 1 & Chest & 11025.61 & 10998.49 & 11028.51 & 7.74 & 7.75 & 7.74 \\
2 & Brain & 12352.67 & 12318.60 & 12291.03 & 7.25 & 7.26 & 7.27 \\
3 & MR & 11484.95 & 11495.07 & 11425.25 & 7.56 & 7.56 & 7.58 \\
\hline
\end{tabular}

Table 10 Comparison of average MSE and PSNR values

\begin{tabular}{llcc}
\hline S. no. & Algorithms & MSE values & PSNR values \\
\hline 1 & Proposed & 11017.53 & 7.74 \\
2 & [44]-Lena & 4859.03 & 11.30 \\
3 & [44]-Baboon & 6399.05 & 10.10 \\
4 & [44]-Pepper & 7274.44 & 9.55 \\
\hline
\end{tabular}




$$
\operatorname{PSNR}=10 \times \log _{10}\left(\frac{255 \times 255}{\mathrm{MSE}}\right)
$$

where MSE is the mean squared error value. Lower value PSNR indicates that there is a significant difference between the original plaintext image and its encrypted counterpart, which is desirable for an encryption algorithm. Here we applied the PSNR test on all three medical images for three different directions. The average PSNR value (in all directions) for chest X-ray, brain, and MR medical images were 7.74, 7.26, and 7.57 respectively, as shown in Table 9. These results are even better than the values obtained by [44], as shown in Table 10. The lower values we achieved intimate that our designed cryptosystem outperforms existing cryptosystems significantly.

\subsection{Entropy Analysis}

The most critical parameter for the measurement of unpredictability and randomness is entropy as first introduced by Claude E. Shannon in 1948 [16]. According to Shannon, information entropy describes the degree of uncertainty present in any communication system. The mathematical formula for the calculation of information entropy is:

$$
H(m)=\sum_{i=0}^{2^{N}-1} p\left(m_{i}\right) \log \frac{1}{p\left(m_{i} .\right)}
$$

where $p\left(m_{i}\right)$ represents the probability of $m_{i}$. and $N$ shows the number of bits representing $m_{i}$. If we consider a random source that can generate $2^{K}$ symbols, the entropy value will be $K$. As we know that a gray-scale image has $2^{8}$ grey levels, so the entropy value should ideally be eight, but practically it is a value less than eight. The calculated results are benchmarked for chest, brain, and MR in Tables 11, 12 and 13 respectively. The results are compared to those achieved by [45] Sun's scheme, [45] Baptisa's scheme, [45] Wong's scheme , and [45] Xiang's scheme as shown in Table 14. The calculated entropy values generated by our proposed cryptosystem are higher than those obtained from existing schemes.

\subsubsection{Time Complexity}

An efficient cryptosystem should take up minimum resources and time during its execution process. To analyze the time and computational complexity needed here, our proposed algorithm is compared with those offered by [3, 3, 7, 7, 23, 23]. The host system used for this timing complexity analysis had the following specifications: Acer TMP-455-MG computer with intel core i5 CPU and 8GB RAM. The computer was also equipped with AMD Radeon graphics card (HD 8750M) and the Matlab 2013(a) simulation tool was used to perform the experiments. The time complexity results in Table 15 demonstrate the higher

Table 11 Information entropy test for chest image along three direction

\begin{tabular}{lllll}
\hline S. no & Name & Along X & Along Y & Along Z \\
\hline 1 & Actual entropy & 7.0899 & 7.0899 & 7.0899 \\
2 & Ideal entropy & 8.0000 & 8.0000 & 8.0000 \\
3 & Ciphered & 7.9994 & 7.9993 & 7.9993 \\
\hline
\end{tabular}


Table 12 Information entropy test for MR image along three direction

Table 13 Information entropy test for brain image along three direction

Table 14 The comparison of entropy values

Table 15 Time complexity of the proposed scheme and its comparison

\begin{tabular}{lllll}
\hline S. no & Name & Along X & Along Y & Along Z \\
\hline 1 & Actual entropy & 7.0634 & 7.0634 & 7.0634 \\
2 & Ideal entropy & 8.0000 & 8.0000 & 8.0000 \\
3 & Ciphered & 7.9986 & 7.9986 & 7.9986 \\
\hline
\end{tabular}

\begin{tabular}{lllll}
\hline S. No & Name & Along X & Along Y & Along Z \\
\hline 1 & Actual entropy & 7.2399 & 7.2399 & 7.2399 \\
2 & Ideal entropy & 8.0000 & 8.0000 & 8.0000 \\
3 & Ciphered & 7.9993 & 7.9993 & 7.9993 \\
\hline
\end{tabular}

\begin{tabular}{lll}
\hline S. no. & Algorithms & Entropy values \\
\hline 1 & Chest (all directions) & 7.9995 \\
2 & [45], Sun's scheme & 7.9965 \\
3 & [45], Baptisa's scheme & 7.9260 \\
4 & [45], Wong's scheme & 7.9690 \\
5 & [45], Xiang's scheme & 7.9950 \\
\hline
\end{tabular}

\begin{tabular}{lll}
\hline S. no. & Images & $\begin{array}{l}\text { Calcu- } \\
\text { lated } \\
\text { time }(\mathrm{s})\end{array}$ \\
\hline $\begin{array}{ll}\text { Time complexity of the proposed } \\
\text { scheme and its comparison 1 }\end{array}$ & Proposed Scheme & 1.53 \\
2 & [3]-Pepper & 3.68 \\
3 & [7]-pepper & 2.76 \\
4 & [23]-pepper & 2.17 \\
5 & [3]-Lena & 3.23 \\
6 & [7]-Lena & 2.25 \\
7 & [23]-Lena & 2.14 \\
\hline
\end{tabular}

agility of the proposed scheme with $1.53 \mathrm{~s}$ of time required to encrypt a medical image, which is much lower than the time required by existing techniques.

\section{Conclusion}

In this paper, we highlighted the need for a secure encryption technique to protect confidential information in medical images. An enhanced image encryption scheme is proposed that combines chaos theory with Brownian motion (BM) and Chen's chaotic system (CCS) 
to achieve the desired level of security in storage systems of hospitals and medical centers. The proposed system achieves confusion through two-dimensional Henon chaotic map (HCM), whereas diffusion is obtained using BM and CCS. Furthermore, the reliability and security of the proposed system are analyzed and compared with existing techniques using the following parameters. The NIST and entropy measures are obtained through randomness test, the consistency and variance through histogram examination, and the pixel similarity using a coefficient of correlation. Other performance analysis parameters include energy, contrast, homogeneity, mean square error, peak to signal noise ratio, number of pixels changing the rate, unified average changing intensity, and computational complexity. The results show that the proposed system outperform existing image encryption systems in terms of higher security. In addition, the proposed system requires less computational resources and, at the same time, offer fast processing making it suitable for application in real-time encryption. As a future direction, the proposed encryption scheme can be modified in order to encrypt other media formats including audio and video.

Open Access This article is licensed under a Creative Commons Attribution 4.0 International License, which permits use, sharing, adaptation, distribution and reproduction in any medium or format, as long as you give appropriate credit to the original author(s) and the source, provide a link to the Creative Commons licence, and indicate if changes were made. The images or other third party material in this article are included in the article's Creative Commons licence, unless indicated otherwise in a credit line to the material. If material is not included in the article's Creative Commons licence and your intended use is not permitted by statutory regulation or exceeds the permitted use, you will need to obtain permission directly from the copyright holder. To view a copy of this licence, visit http://creativecommons.org/licenses/by/4.0/.

\section{References}

1. Agarwal, S. (2018). Secure image transmission using fractal and 2D-chaotic map. Journal of Imaging, 4(1), 17.

2. Ahmad, H. (2016). A secure image encryption scheme based on chaotic maps and affine transformation. Multimedia Tools and Applications, 75(21), 13951-13976.

3. Ahmad, J., \& Hwang, S. O. (2016). A secure image encryption scheme based on chaotic maps and affine transformation. Multimedia Tools and Applications, 75(21), 13951-13976.

4. Ahmad, J., Khan, M. A., Hwang, S. O., \& Khan, J. S. (2017). A compression sensing and noise-tolerant image encryption scheme based on chaotic maps and orthogonal matrices. Neural Computing and Applications, 28(1), 953-967.

5. Ahmad, J., Larijani, H., Emmanuel, R., \& Mannion, M., et al. (2018). Secure occupancy monitoring system for IoT using lightweight intertwining logistic map. In 2018 10th computer science and electronic engineering (CEEC) (pp. 208-213). IEEE.

6. Ahmad, J., Masood, F., Shah, S. A., Jamal, S. S., \& Hussain, I. (2020). A novel secure occupancy monitoring scheme based on multi-chaos mapping. Symmetry, 12(3), 350.

7. Ahmed, F., Anees, A., Abbas, V. U., \& Siyal, M. Y. (2014). A noisy channel tolerant image encryption scheme. Wireless Personal Communications, 77(4), 2771-2791.

8. Ali, T. S., \& Ali, R. (2020). A novel medical image signcryption scheme using TLTS and Henon chaotic map. IEEE Access, 8, 71974-71992.

9. Allah, M. F., \& Eid, M. (2020). Chaos based 3D color image encryption. Ain Shams Engineering Journal, 11(1), 67-75.

10. Bashir, I., Ahmed, F., Ahmad, J., Boulila, W., \& Alharbi, N. (2019). A secure and robust image hashing scheme using gaussian pyramids. Entropy, 21(11), 1132.

11. Behnia, S., Akhshani, A., Mahmodi, H., \& Akhavan, A. (2008). A novel algorithm for image encryption based on mixture of chaotic maps. Chaos, Solitons \& Fractals, 35(2), 408-419.

12. Chen, G., \& Ueta, T. (1999). Yet another chaotic attractor. International Journal of Bifurcation and chaos, 9(07), 1465-1466. 
13. Dai, Y., Wang, H., Zhou, Z., \& Jin, Z. (2016). Research on medical image encryption in telemedicine systems. Technology and Health Care, 24(s2), S435-S442.

14. Elkandoz, M. T., Alexan, W., \& Hussein, H. H. (2019). Logistic sine map based image encryption. In 2019 Signal processing: Algorithms, architectures, arrangements, and applications (SPA) (pp. 290295). IEEE.

15. Gao, T., \& Chen, Z. (2008). A new image encryption algorithm based on hyper-chaos. Physics Letters A, 372(4), 394-400.

16. Guizzo, E. M. (2003). The essential message: Claude Shannon and the making of information theory. $\mathrm{Ph} . \mathrm{D}$. Thesis, Massachusetts Institute of Technology.

17. Hénon, M. (1976). A two-dimensional mapping with a strange attractor. In The theory of chaotic attractors (pp. 94-102). Springer.

18. Huang, V. L., Zhao, S. Z., Mallipeddi, R., \& Suganthan, P. N. (2009). Multi-objective optimization using self-adaptive differential evolution algorithm. In 2009 IEEE congress on evolutionary computation (pp. 190-194). IEEE.

19. Kaur, M., \& Kumar, V. (2018). A comprehensive review on image encryption techniques. Archives of Computational Methods in Engineering 1-29.

20. Khan, F. A., Ahmed, J., Khan, J. S., Ahmad, J., \& Khan, M. A. (2017). A novel substitution box for encryption based on Lorenz equations. In 2017 International conference on circuits, system and simulation (ICCSS) (pp. 32-36). IEEE.

21. Khan, M., \& Masood, F. (2019). A novel chaotic image encryption technique based on multiple discrete dynamical maps. Multimedia Tools and Applications, 78(18), 26203-26222.

22. Khan, M., Masood, F., \& Alghafis, A. (2019). Secure image encryption scheme based on fractals key with Fibonacci series and discrete dynamical system. Neural Computing and Applications $1-21$.

23. Khan, M., Masood, F., Alghafis, A., Amin, M., \& Batool Naqvi, S. I. (2019). A novel image encryption technique using hybrid method of discrete dynamical chaotic maps and Brownian motion. PLOS ONE, 14(12), e0225031.

24. Li, C., \& Chen, G. (2004). Chaos in the fractional order Chen system and its control. Chaos, Solitons \& Fractals, 22(3), 549-554.

25. Liu, H., \& Wang, X. (2010). Color image encryption based on one-time keys and robust chaotic maps. Computers \& Mathematics with Applications, 59(10), 3320-3327.

26. Mansouri, A., \& Wang, X. (2020). A novel one-dimensional sine powered chaotic map and its application in a new image encryption scheme. Information Sciences, 520, 46-62.

27. Masood, F., Ahmad, J., Shah, S. A., Jamal, S. S., \& Hussain, I. (2020a). A novel hybrid secure image encryption based on Julia set of fractals and 3D Lorenz chaotic map. Entropy, 22(3), 274.

28. Masood, F., Boulila, W., Ahmad, J., Sankar, S., Rubaiee, S., Buchanan, W. J., et al. (2020b). A novel privacy approach of digital aerial images based on Mersenne twister method with DNA genetic encoding and chaos. Remote Sensing, 12(11), 1893.

29. Özkaynak, F. (2019). Chaos based substitution boxes as a cryptographic primitives: Challenges and opportunities. Chaotic Modeling and Simulation, 1, 49-57.

30. Özkaynak, F. (2020). On the effect of chaotic system in performance characteristics of chaos based s-box designs. Physica A: Statistical Mechanics and its Applications, 550, 124072.

31. Pareek, N. K., Patidar, V., \& Sud, K. K. (2006). Image encryption using chaotic logistic map. Image and Vision Computing, 24(9), 926-934.

32. Pavithra, V., \& Chandrasekaran, J. (2021). Developing security solutions for telemedicine applications: medical image encryption and watermarking. In Research anthology on telemedicine efficacy, adoption, and impact on healthcare delivery (pp. 612-631). IGI Global.

33. Qayyum, A., Ahmad, J., Boulila, W., Rubaiee, S., Masood, F., Khan, F., \& Buchanan, W. J. et al. (2020). Chaos-based confusion and diffusion of image pixels using dynamic substitution. IEEE Access.

34. Radwan, A. G., AbdElHaleem, S. H., \& Abd-El-Hafiz, S. K. (2016). Symmetric encryption algorithms using chaotic and non-chaotic generators: A review. Journal of Advanced Research, 7(2), 193-208.

35. Rhouma, R., Meherzi, S., \& Belghith, S. (2009). Ocml-based colour image encryption. Chaos, Solitons \& Fractals, 40(1), 309-318.

36. Sasikaladevi, N., Geetha, K., Sriharshini, K., \& Aruna, M. D. (2020). H3-hybrid multilayered hyper chaotic hyper elliptic curve based image encryption system. Optics \& Laser Technology, 127, 106173.

37. Seyedzadeh, S. M., Norouzi, B., Mosavi, M. R., \& Mirzakuchaki, S. (2015). A novel color image encryption algorithm based on spatial permutation and quantum chaotic map. Nonlinear Dynamics, 81(1-2), 511-529.

38. Shannon, C. E. (1949). Communication theory of secrecy systems. Bell System Technical Journal, $28(4), 656-715$. 
39. Shao, Z., Liu, X., Yao, Q., Qi, N., Shang, Y., \& Zhang, J. (2020). Multiple-image encryption based on chaotic phase mask and equal modulus decomposition in quaternion gyrator domain. Signal Processing: Image Communication, 80, 115662.

40. Tsafack, N., Kengne, J., Abd-El-Atty, B., Iliyasu, A. M., Hirota, K., \& Abd EL-Latif. A. A. (2020). Design and implementation of a simple dynamical 4-D chaotic circuit with applications in image encryption. Information Sciences, 515, 191-217.

41. Tutueva, A. V., Nepomuceno, E. G., Karimov, A. I., Andreev, V. S., \& Butusov, D. N. (2020). Adaptive chaotic maps and their application to pseudo-random numbers generation. Chaos, Solitons \& Fractals, 133, 109615.

42. Wang, S., Wang, C., \& Xu, C. (2020a). An image encryption algorithm based on a hidden attractor chaos system and the Knuth-Durstenfeld algorithm. Optics and Lasers in Engineering, 128, 105995.

43. Wang, X., Wang, Y., Zhu, X., \& Luo, C. (2020b). A novel chaotic algorithm for image encryption utilizing one-time pad based on pixel level and DNA level. Optics and Lasers in Engineering, 125, 105851 .

44. Younas, I., \& Khan, M. (2018). A new efficient digital image encryption based on inverse left almost semi group and Lorenz chaotic system. Entropy, 20(12), 913.

45. Zhang, G., \& Liu, Q. (2011). A novel image encryption method based on total shuffling scheme. Optics Communications, 284(12), 2775-2780.

46. Zhou, M., \& Wang, C. (2020). A novel image encryption scheme based on conservative hyperchaotic system and closed-loop diffusion between blocks. Signal Processing, 171, 107484.

Publisher's Note Springer Nature remains neutral with regard to jurisdictional claims in published maps and institutional affiliations.

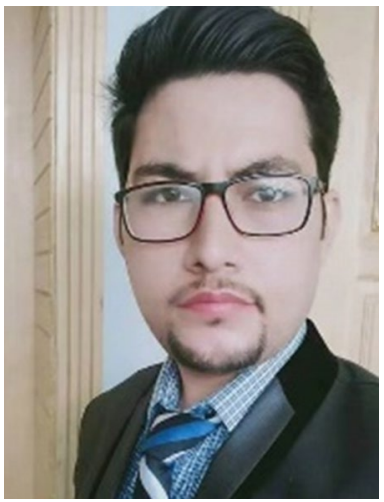

Fawad Masood received B.S. and M.S. degrees in Electrical Engineering from CECOS University, Pakistan in July, 2015 and Institute of Space Technology, Islamabad, Pakistan in Feb, 2019, respectively. Currently, he is working in the area of Cybersecurity under the supervision of Dr Jawad Ahmad, Edinburgh Napier University, UK. Fawad has numerous research publications in the field of image encryption. His research area includes cybersecurity, information security, coding and cryptography, chaos theory and its application, fractals based image encryption, non-linear dynamical systems and data communication.

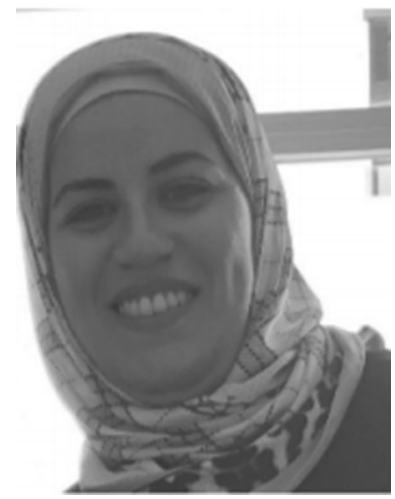

Maha Driss received the Engineering degree (Hons.) in computer science, the M.Sc. degree from the National School of Computer Science (ENSI), University of Manouba, Tunisia, in 2006 and 2007, respectively, and the Ph.D. degree conjointly from the University of Manouba, Tunisia, and University of Rennes 1, France, in 2011. From 2012 to 2015, she was an Assistant Professor in computer science with the National Higher Engineering School of Tunis, University of Tunis, Tunisia. She is currently an Assistant Professor of computer science with the IS Department, College of Computer Science and Engineering, Taibah University, Saudi Arabia. She is also a Senior Researcher with the RIADI Laboratory, University of Manouba. Her primary research interests include software engineering, Web service computing, distributed systems, IoT, and artificial intelligence. 

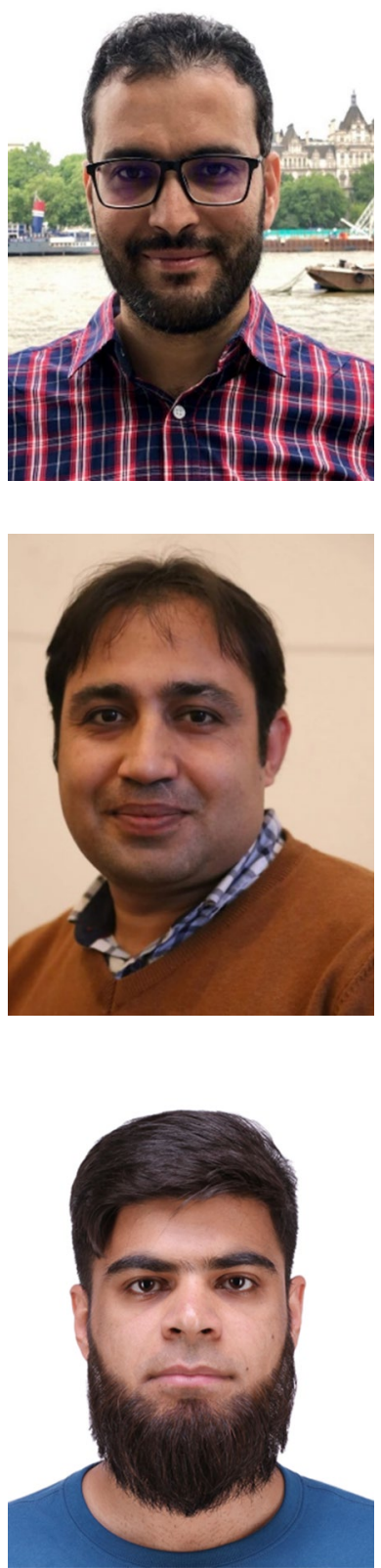

Wadii Boulila received the Eng. degree (Hons.) in computer science from the Aviation School of Borj El Amri, in 2005, the M.Sc. degree from the National School of Computer Science (ENSI), University of Manouba, Tunisia, in 2007, and the Ph.D. degree conjointly from ENSI and Telecom-Bretagne, University of Rennes 1, France, in 2012. He is currently an Assistant Professor of computer science with the IS Department, College of Computer Science and Engineering, Taibah University, Medina, Saudi Arabia. He is also a Senior Researcher with the RIADI Laboratory, University of Manouba, and an Associate Researcher with the ITI department, University of Rennes 1. His primary research interests include big data analytics, deep learning, data mining, artificial intelligence, cybersecurity, uncertainty modeling, and remote sensing images. He has served as the Chair, a Reviewer, and a TPC Member for many leading international conferences and journals.

Jawad Ahmad is an experienced researcher with more than ten years of cutting-edge research and teaching experience in prestigious institutes, including Edinburgh Napier University, U.K., Glasgow Caledonian University, U.K., Hongik University, South Korea, and HITEC University, Taxila, Pakistan. He has co-authored more than 60 research articles, in international journals and peer-reviewed international conference proceedings. He has taught various courses both at Undergraduate (UG) and Postgraduate (PG) levels during his career. He regularly organizes timely special sessions and workshops for several flagship IEEE conferences. His research interests include cybersecurity, machine learning and deep learning, chaos theory and multimedia encryption.

Sadaqat Ur Rehman received his B.Sc. degree from the Department of Computer Systems Engineering, University of Engineering and Technology Peshawar and M.Sc. degrees from the Department of Electrical Engineering, Sarhad University of Science and IT in 2011 and 2014, respectively. Dr. Sadaqat ur Rehman pursued his Ph.D. degree (Sept. 2015-Jun. 2019) with the Tsinghua National Laboratory for Information Science and Technology, Tsinghua University, Beijing, China. He worked as Artificial Intelligence Engineer in Schlumberger (Beijing Geoscience Centre) from November 2019 to June 2020, where he developed different Deep Learning/Machine Learning models for Drilling Dynamics Computation Engine. He is currently working as Assistant Professor with the Department of Computer Science, Namal Institute-Mianwali, Pakistan. He is also a visiting lecturer/researcher at the Department of Computer Science, Beijing University of Technology, Beijing, China. He has produced a world leading research activity in the fields of data science, machine learning, intelligent systems (with emphasis on artificial neural networks), semantic multimedia analysis, optimization and affective computing. He has published 28 papers in international journals and 08 papers in proceedings of international conferences. His research has been highly referenced (about 300+ citations with an h-index of 10 in Google Scholar). Also, he is co-chair, TPC member and reviewer of prestigious international conferences and journals including, BMLI 2020, Smarttech 2020, CSAE 2018, 2019, 2020, IEEE Access, Neurocomputing, IEEE TCSVT, Scientific Reports-Nature, Soft Computing, Signal Processing. 

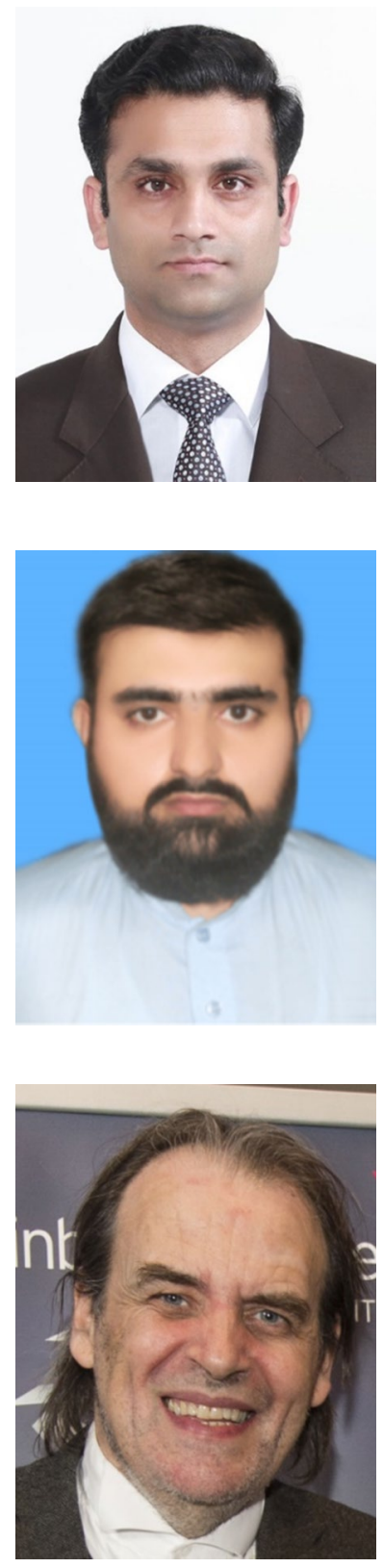

Sana Ullah Jan is an experienced researcher with more than 6 years of cutting-edge research and teaching experience in prestigious institutes including the University of the West of Scotland, the University of Ulsan (South Korea) and the University of Lahore Islamabad Campus (Pakistan). He is currently enrolled as Post-doctoral Research Fellow at the Center of Affective and Human Computing for Smart Environment at the school of computing, engineering and physical sciences, University of the West of Scotland since September 2020. He has (co) authored 9 journal papers and 7 peer-reviewed international conference proceedings. His research area is related to the Artificial Intelligence or Machine Learning-based cyber security and privacy in the Internet-of-Things, Cyber Physical Systems and eHealth. He has taught various courses both at Undergraduate (UG) and Postgraduate (PG) levels during my career. He is an invited reviewer for leading high-impact journals (reviewed $30+$ journals to date).

Abdullah Qayyum was born in Mardan, Khyber Pakhtunkhwa, Pakistan, in August 1996. He received his BS degree in Electrical Engineering (major in communication) from the University of Engineering and Technology (UET) Peshawar, Pakistan, in 2019. His research interests include wireless communication, $5 \mathrm{G}$, antennas, cybersecurity, and image encryption.

William J. Buchanan is currently a Professor with the School of Computing, Edinburgh Napier University. He was awarded an OBE in the Queen's Birthday awards, in June 2017. He also leads the Centre for Distributed Computing, Networks, and Security and The Cyber Academy, and works in the areas of security, cloud security, Web-based infrastructures, e-crime, cryptography, triage, intrusion detection systems, digital forensics, mobile computing, agent-based systems, and security risk. He has one of the most extensive academic sites in the World and is involved in many areas of novel research and teaching in computing. He has published more than 27 academic books, and more than 250 academic research articles, along with several awards for excellence in knowledge transfer, and for teaching. He was named as one of the Top 100 people for Technology in Scotland from 2012 to 2017. Recently, he was included in the FutureScot Top 50 Scottish Tech People Who Are Changing The World. He is a Fellow of the BCS and the IET. 